\title{
Assembly status transition offers an avenue for allosteric activity modulation of a supramolecular enzyme
}

3

Yao Chen ${ }^{\mathrm{a}^{*}}$, Weiya $\mathrm{Xu}^{\mathrm{a}^{*}}$, Shuwei $\mathrm{Yu}^{\mathrm{b}^{*}}$, Kang Nic, Guangbiao, She ${ }^{\mathrm{b}}$, Xiaodong Yec, Qiong Xingd\#, Jian Zhaob\#, Chengdong Huanga\#

${ }^{*}$ These authors contributed equally \# Corresponding author: Qiong Xing (qiongxingnmr@hubu.edu.cn); Jian Zhao

(jzhao2@qq.com); Chengdong Huang (huangcd@ustc.edu.cn)

Affiliations:

a: Ministry of Education Key Laboratory for Membrane-less Organelles \& Cellular Dynamics, Hefei National Laboratory for Physical Sciences at the Microscale, School of Life Sciences, Division of Life Sciences and Medicine, University of Science and Technology of China, 230027 Hefei, P.R. China

b: State Key Laboratory of Tea Plant Biology and Utilization, College of Tea and Food Science and Technology, Anhui Agricultural University, Hefei, 230036, China.

c: Hefei National Laboratory for Physical Sciences at the Microscale, Department of Chemical Physics, University of Science and Technology of China, Hefei, Anhui 230026, China

d: State Key Laboratory of Biocatalysis and Enzyme Engineering, Hubei Collaborative Innovation Center for Green Transformation of Bio-Resources, Hubei Key Laboratory of Industrial Biotechnology, School of Life Sciences, Hubei University, Wuhan, China. 


\section{$31 \quad$ Abstract}

32 Nature has evolved many supramolecular proteins assembled in certain, sometimes

33 even seemingly oversophisticated, morphological manners. The rationale behind such

34 evolutionary efforts is often poorly understood. Here we provide atomic-resolution

35 insights into how the dynamic building of a structurally complex enzyme with higher-

36 order symmetry offers amenability to intricate allosteric regulation. We have established

37 the functional coupling between enzymatic activity and protein morphological states of

38 glutamine synthetase (GS), an old multi-subunit enzyme essential for cellular nitrogen

39 metabolism. Cryo-EM structure determination of GS in both the catalytically active and

40 inactive assembly states allows us to reveal an unanticipated self-assembly-induced

41 dynamics-driven allosteric paradigm, in which the remote interactions between two

42 subcomplex entities significantly rigidify the otherwise structurally fluctuating active sites,

43 thereby regulating activity. We further show in vivo evidences that how the enzyme

44 morphology transitions could be modulated by cellular factors on demand. Collectively,

45 our data present an example of how assembly status transition offers an avenue for

46 allosteric modulation, and sharpens our mechanistic understanding of allostery,

47 dynamics, cooperativity, and other complex functional and regulatory properties of

48 supramolecular enzymes. 


\section{Introduction}

55 Recent studies have evidenced that only a small portion of proteins function in isolation

56 in cells whereas the majority is assembled into complexes through protein-protein

57 interactions with identical or different protein subunit(s) ${ }^{1}$. The rationale behind such an

58 evolutionary selection has been the subject of considerable speculation; proposals for

59 the advantages associated with a multimeric-units complex instead of a long single

60 polypeptide chain include better error control in synthesis, greater coding and folding

61 efficiency, and possibility of allosteric regulation ${ }^{2}$. Morphologically speaking, many

62 protein complexes especially homomeric ones adopt a symmetric spatial arrangement,

63 either cyclic $\left(C_{n(n>1)}\right)$ or dihedral $\left(D_{n}(n>1)\right)$ symmetry, characterized by a rotational

64 symmetry or two orthogonal symmetry axes, respectively. In contrast to the cyclic

65 complexes which evolve in one step (e.g. C1 $\rightarrow$ C5), evolution of dihedral complexes

66 takes place in multiple steps (e.g. C1 $\rightarrow$ C5 $\rightarrow$ D5) ${ }^{3}$, and adds another layer of structural

67 complexity. Intriguingly, pioneering studies have revealed many supramolecular

68 enzymes organized in dihedral symmetry, with subcomplex entities in cyclic symmetry

69 holding, at least outwardly, multiple integral active sites. Thus a fundamental question

70 arises here is that why nature builds these protein complexes with a seemingly

71 oversophisticated quaternary design, if the subcomplexes alone possess complete

72 elements for action? In other words, is the extra assembly step, e.g. C5 $\rightarrow$ D5, is a futile

73 evolutionary effort for these supramolecular protein complexes? 
74 One such an example is glutamine synthetases (GSs) (EC 6.3.1.2), one of the most

75 ancient functioning enzymes in existence and a central enzyme in nitrogen metabolism

76 of all living organisms, catalyzing the formation of glutamine by condensation of

77 glutamate with ammonia using ATP as an energy source ${ }^{4,5}$. Three classes of GS

78 enzymes have been identified in different organisms, namely, GSI, GSII and GSIII.

79 Decades of studies have established a striking notion that all three classes of GS

80 enzymes, despite of dramatic differences in amino acid sequences and protein sizes,

81 share quaternary geometry in dihedral symmetry assembled with two oligomeric rings ${ }^{6-}$

$82{ }^{13}$. Considering that the active sites of GS are located at the clefts formed between two

83 neighboring protomers within the same ring and distal to the ring-ring interface, each

84 isolated GS subcomplex ring holds multiple integral catalytic sites ${ }^{4,14}$. The functional

85 demand for this evolutionary conservation, the quaternary organization of GS with

86 dihedral symmetry, remains elusive.

87 Here we sought out to explore the functional link between the oligomeric conformation

88 and catalysis activity, and mechanistically justify the seemingly oversophisticated

89 assembly design in this supramolecular enzyme. Our results unveil a previously

90 uncharacterized dynamics-driven allostery mechanism induced by assembly status

91 transition of GS, and present an example that how a particular quaternary geometry

92 selectively defines the oligomer dynamics congruent with required allosteric activities.

93 We further show in vivo evidence how this regulatory machinery is elegantly utilized by

94 the cell to meet the ever-changing metabolic needs. The functional implications of these

95 findings are discussed.

96 Results 
97 Two highly conserved GSIls demonstrate distinct quaternary structure 98 organization propensities

99 With the aim of clarifying the functional role of dihedral symmetry in GS functions, we

100 first carried out a quest for GSs that share a high degree of sequence conservation, but

101 demonstrate distinct quaternary structural assembly properties. We make use of the

102 weak ring-ring interaction of GSII, a prominent structural difference between the type I

103 and type II GSs ${ }^{11-13}$, and seek GSII variants from different species with amino acid

104 variations mainly occurring at the pentamer interface, which may thus present disparate

105 decamer-forming propensities. We built model structures for the candidate GSIls based

106 on the crystal structure of the maize GSII (pdb code:2D3B) and analyzed the amino acid

107 variations in the context of model structures. Primary structure analysis reveals GSIls

108 from the plants of Camellia sinensis (CsGSIb) and Glycine max (GmGS $\beta 2$ ) share an

109 overall very high sequence homology ( 90\% identical and $\sim 97 \%$ conserved) and

110 absolutely conserved substrate-binding and catalytic sites (Fig. 1a), with, however, a

111 significant portion of amino acid variations clustered at the interface between two

112 pentamer rings (Fig. 1a and 1b). We then recombinantly expressed both CsGSIb and

113 GmGS $\beta 2$ in E. coli and purified these two GSIl homologs. To assess the oligomerization

114 status, we performed size-exclusion chromatography (SEC) coupled to both multi-angle

115 light scattering (MALS) and quasi-elastic light scattering (QELS). Despite sharing an

116 overall highly conserved amino acid sequence, CsGSIb and GmGS $\beta 2$ exhibit distinct

117 quaternary structural properties. MALS analysis shows the GSIl from Glycine max being

118 largely a homogeneous decamer in solution (Fig. 1c). In contrast, under the same

119 condition the majority fraction ( 82\%) of CsGSIb adopts a pentameric configuration, 
120 along with a minor fraction ( 18\%) being decameric (Fig. 1d). We further show that

121 CsGSIb exists in pentamer-decamer dynamic equilibrium in solution and a mixture of

122 electrostatic and hydrophobic interactions is responsible for attaching of two pentameric

123 rings; whereas substrates or ligands show no appreciable effect on the decamer-

124 forming properties (see Supplementary data for details).

125 We further employed negative-stain electron microscopy (EM) to directly visualize the 126 distinct ring-ring packing propensities for CsGSIb and GmGSß2. Two-dimensional (2D)

127 class averages revealed that GmGSß2 forms homogeneous, double stacked-ring 128 shaped particles (Fig. 1e), in line with the decameric organization pattern previously 129 reported for other GSII species ${ }^{11-13}$. In contrast, CsGSIb adopted a mixture of two 130 quaternary structural modes: detached pentamers (Fig. 1f) and decamers composed of 131 two stacked pentamer rings (Fig. 1g), consistent with the above MALS analysis result 132 (Fig. 1d).

133 Analytical ultracentrifugation (AUC) was carried out to quantitively assess the

134 thermodynamic parameters of CsGSIb pentamer-decamer transition, which 135 demonstrated two species for CsGSIb in solution with molecular weights of $191 \mathrm{kDa}$ 136 and $395 \mathrm{kDa}$ (Fig. 2a), corresponding to the pentameric and decameric configurations, 137 respectively. Sedimentation profiles at various protein concentrations were analyzed 138 and a global analysis of the data at each protein concentration yielded a pentamer139 pentamer dissociation constant $(\mathrm{Kd})$ of $0.27 \pm 0.06 \mu \mathrm{M}$ at room temperature. It is 140 noteworthy that the dissociation constant within the sub-micromolar range allows 141 CsGSIb to predominantly exist as isolated pentamers under the concentration assayed 
142 for enzymatic activities, laying a solid foundation for probing the functional role of the

143 decamer formation in modulating the enzyme activity of GS.

144 GSIls in different assembly states demonstrate distinct enzymatic activities

145 We next sought to compare the glutamine synthesis activity of these two highly

146 conserved GSIls. As shown in Fig. 3b and Fig. S2a, when supplied with ammonium

147 chloride, the stable decamer-adopting GmGS 22 demonstrated significant GS activities.

148 Further steady-state kinetic measurements yielded turnover numbers ( $\left.\mathrm{k}_{\text {cat }}\right)$ and

149 Michaelis constants $\left(\mathrm{K}_{\mathrm{m}}\right)$ of $\sim 12.8 \mathrm{~min}^{-1}$ and $\sim 44 \mu \mathrm{M}$, respectively (Fig. 3c). In sharp

150 contrast, CsGSIb, for which the majority protein exists as discrete pentamers under the

151 condition assayed, only demonstrated basal activity (Fig. 3b and Fig. S3a), consistent

152 with previous observations that the isolated single-ringed GSII species is nonfunctional

15315,16 . These observations raised the question as to whether the drastic disparity in

154 catalytic activities for these two highly conserved GSIl could be attributed to their

155 dramatically different propensities for formation of a double-ringed architecture. If the

156 above proposal holds true, a positive concentration-dependent cooperation of enzyme

157 activity would be expected as increase in the concentration of CsGSIb favors decamer

158 assembly (Fig. S1a). Indeed, five times increase in the concentration of CsGSIb

159 assayed (from $1 \mu \mathrm{M}$ to $5 \mu \mathrm{M}$ ) resulted in $\sim 30$ folds increase in activity, i.e. $\sim 6$ folds

160 activity increase per unit of enzyme, displaying a significant concentration-dependent 161 simulation effect (Fig. S2b).

162 Catalytic switching of CsGSIb through oligomeric states interconversion

163 To further validate the above proposal, we performed mutagenesis to CsGSIb, aiming

164 to convert its unstable pentamer-decamer equilibrium state to a stable decamer and 
165 then evaluate the impact on catalytic activity. According to the amino acid sequence of

166 GmGSß2, three CsGSIb mutants, i.e. EVK138DIQ, I143L and Y150F, respectively,

167 were selected, expressed and subsequently purified as the wild type (WT) protein. We

168 then performed SEC-MALS measurements to evaluate mutational effects on their

169 oligomeric states. As shown in Fig. $2 \mathrm{dx}$ and $2 \mathrm{f}$, both mutations of $1143 \mathrm{~L}$ and

170 EVK138DIQ led to a drastic shift in the oligomerization equilibrium towards decamer

171 assembly, causing significant increase in the distribution of decameric state from $\sim 18 \%$

172 in the WT-CsGSIb (Fig. 1d) to $>95 \%$ and $\sim 72 \%$, respectively. These observations

173 confirmed that both mutations introduced at the interface, albeit largely conservative,

174 dramatically fortified the decamer edifice assembly. In contrast, substitution of the

175 tyrosine at the residue 150 with a phenylalanine showed no appreciable change in its

176 quaternary organization mode (Fig. S3a), suggesting no critical role for the residue

177 Y150 in maintaining two CsGSIb pentameric ring subcomplexes attached. We 178 employed AUC analysis to measure the ring-ring dissociation constant for mutants of

179 I143L and EVK138DIQ. As demonstrated in Fig. 2e and 2f, replacement of I143 or EVK

180 at residues 138-140 with leucine or DIQ yielded ring-ring disassociation constants of

$181 \sim 0.01$ or $\sim 0.04 \mu \mathrm{M}$, respectively, i.e., $\sim 27$ or $\sim 7$ folds of increase in the ring-ring binding

182 affinity compared with that of WT-CsGSIb. The Gibbs energy changes upon mutation

$183(\Delta \Delta \mathrm{G})$ for $1143 \mathrm{~L}$ and EVK138DIQ were calculated to be $-8.1 \mathrm{~kJ} / \mathrm{mol}$ and $-4.7 \mathrm{~kJ} / \mathrm{mol}$, 184 respectively.

185 We next set out to investigate the resulting impacts on their enzymatic activities. As 186 shown in Fig. 2h-2j, both mutations of I143L and EVK138DIQ caused dramatic increase 187 in catalytic activity of $\sim 76$ and $\sim 64$ folds, respectively. As all mutated amino acids are 
188 distal to either the catalytic site or substrate binding regions with distances $>20 \AA$ and

189 hence are unlikely to be directly involved in catalytic reaction, we infer that the

190 stimulations of the enzymatic activity of CsGSlb upon residue perturbations are

191 attributed to allosteric effects induced by remote contacts between two pentamer rings.

192 As expected, the mutation of Y150F, which did not alter pentamer-decamer equilibrium

193 of CsGSIb (Fig. S3a), showed no noticeable change in enzymatic activity (Fig. S3b).

194 Structural basis for dynamics-driven allostery of GSII

195 To elucidate the allosteric mechanism of how the interactions between two GSII 196 pentameric rings remotely trigger enzymatic activity, we next employed single-particle 197 cryo-EM imaging technique and first determined the structures of GmGS $\beta 2$ decamer, as

198 well as that of the CsGSIb that adopts decameric configuration (thereafter named as 199 CsGSIb ${ }^{\text {Dec }}$ ). 3D classifications of 104717 and 43876 particles for GmGS $\beta 2$ and 200 CsGSIb ${ }^{\text {Dec }}$, respectively, revealed that both molecules were arranged in D5 symmetry 201 with two pentameric rings stacked in a head-to-head manner (Fig. 3a and 3b), a 202 strikingly conserved structural feature that have been widely observed for other type II 203 GS species ${ }^{11-13}$. Refinement of the GmGS 32 and CsGSlb ${ }^{\text {Dec }}$ structures yielded maps 204 with an average resolution of $2.9 \AA$ and $3.3 \AA$, respectively, with literally identical 205 dimensions of $115 \AA$ × $115 \AA$ x $95 \AA$. As expected from the very high conservation in 206 amino acid sequence (Fig. 1a), decamer structures of GmGS 32 and CsGSIb ${ }^{\text {Dec }}$ are very 207 similar to each other, as well as to that of the GSIl of maize (pdb accession number 208 2D3A), as highlighted by the root-mean-square deviation (r.m.s.d.) of $0.66-0.80 \AA$ for 209 328-352 aligned $\mathrm{C}_{\alpha}$ atoms. Structural alignments reveal that the active sites in GmGS $\beta 2$ 210 and CsGSIb ${ }^{D e c}$, as well as that in the maize GSII, are highly conserved (Fig. 3c), 
211 suggesting the catalysis mechanism of these three enzymes, once decamers are

212 formed, are essentially identical. The overall buried inter-ring surfaces for both GmGS $\beta 2$

213 and CsGSIb ${ }^{\text {Dec }}$ amount to $\sim 2000 \AA^{2}$, i.e. approximately only $400 \AA^{2}$ per individual

214 monomer-monomer interaction. This highlights the weakness of the inter-ring contacts,

215 characteristic of type II GS. Indeed, in both structures the inter-ring contacts are

216 established by only a limited number of hydrophobic and polar interactions provided by

217 the residues 136-141 and 146-152 segments of each of the intervening subunits (Fig.

218 S7), which behave as two gear teeth (thereafter named as tooth-1 and tooth-2,

219 respectively) interlocking the two pentameric rings (Fig. 3d-f). This observation is in line

220 with the above result that mutations to tooth-1 resulted in drastic change in oligomeric

221 states behavior (Fig. $2 \mathrm{~d}-2 \mathrm{~g}$ ), and the mixed nature of the inter-ring interactions is

222 consistent with the MALS analysis result of CsGSIb under various buffer conditions (Fig.

223 S1b). Intriguingly, the local structure of the teeth regions that mediate inter-ring

224 interactions remains largely the same in GmGS $\beta 2$ and CsGSIb ${ }^{\text {Dec }}$ (Fig. 3d-3e),

225 suggesting the dramatically different propensities of GmGS $\beta 2$ and CsGSIb for decamer

226 formation are due to the nature of the amino acids involved in inter-ring contacts, rather

227 than the structure. Although the residue of 1143 is not directly involved in inter-ring

228 contact, we argue that its replacement with leucine may stabilize the conformation of

229 tooth-1 via its interaction with the residue of L134, thus playing an important role in

230 stabilizing decamer architecture (Fig. 2d and 2e).

231 In order to elucidate the mechanism of how the pentameric CsGSIb (thereafter named

232 as CsGSIb ${ }^{\text {Pen }}$ ) demonstrates distinct enzymatic properties than CsGSIb ${ }^{\text {Dec }}$ (Fig. 2h), we

233 next determined the structure of CsGSIb ${ }^{\text {Pen }}$. Lowering the sample concentration, which 
234 favored pentamer dissociation (Fig. S1a), allowed us to obtain sufficient number of

235 CsGSIb ${ }^{\text {Pen }}$ particles, which, in turn, enabled us to solve the cryo-EM structure of the

236 inactive single-ringed GSII for the first time. Interestingly, we observed additional class

237 averages in which the two masses of density attributed to the pentameric rings are no

238 longer parallel (Fig. S8). These non-parallel ring particles may reflect intermediate

239 assembly stages in the formation/disruption of the enzyme decamer, again confirming

240 the flexibility of the inter-ring interactions of the type II GS.

241 Unexpected, CsGSIb ${ }^{\text {Pen }}$ exhibited high conformational heterogeneity and 3D particles

242 classification generated three similar structures with the r.m.s.d. ranging from 0.6 to 0.8

$243 \AA$, differing in a few peripheral regions (Fig. S9a). The most striking difference between

$244 \mathrm{CsGSIb}^{\text {Pen }}$ and $\mathrm{CsGSI}^{\mathrm{Dec}}$ is that several regions are missing in the electron density

245 map of all three classifications of $\mathrm{CsGSIb}^{\text {Pen }}$ particles, with only 229 to 255 out of 356

246 residues electron densities in presence. As a result, CsGSIb ${ }^{\text {Pen }}$ demonstrated a

247 decagram-shaped density map with a few regions missing at the rim (Fig. 4a and Fig.

248 S9a), in sharp contrast to a pentagon-shaped map yielded by the CsGSIb ${ }^{\text {Dec }}$ particles

249 (Fig. 4b). For the 229-255 residues that show clear density in CsGSIb ${ }^{\text {Pen }}$ particles, the

250 conformation of each subunit in three CsGSIbPen EM structures, as well as the

251 arrangement pattern, closely resembles that of the CsGSIb ${ }^{\text {Dec }}$, as evidenced by r.m.s.d.

252 in the range of $0.7-1.0 \AA$ (Fig. S9b-d). This result suggest the structures of rigid portion

253 of CsGSIb are not significantly altered upon pentamer association. The density-missing

254 regions include the segments around residues of 110-117, 140-166, and 260-334,

255 among which, the fragment around residues 260-334 is a major component making up

256 an integral catalytic site (Fig. 4c), while the segment of residues 140-166 comprising of 
257 the two gear teeth is responsible for ring-ring interaction (Fig. 3d-f and Fig. 4d). As

258 electron density missing often reflects the conformational heterogeneity arising from

259 internal motions ${ }^{17}$, these observation strongly suggest that the conformation of

260 CsGSIb $^{\text {Pen }}$ active site is highly dynamic, contrasting sharply to the conformationally

261 largely homogeneous CsGSIb ${ }^{\text {Dec }}$. In support this, thermal shift assays show the melting

262 temperature $\left(T_{m}\right)$ of wild-type CsGSIb is significantly lower than that of its mutants of

263 I143L or EVK138DIQ, indicating of structural instability for the pentameric GSII (Fig.

264 S10). We therefore conclude that the dramatically difference in the dynamic property of

265 catalytic sites accounts for the distinct activities of pentameric and decameric CsGSIb.

266 Taken together, our cryo-EM structures allow us to propose a dynamics-driven allosteric

267 mechanism of how the GSIl activity is regulated by changes in oligomeric state: (1) The

268 active sites within isolated CsGSIb $^{\text {Pen }}$ rings are highly disordered and the unstable

269 catalytic environments render it catalytically inactive; (2) Upon stacking of two

270 pentameric rings and formation of a decamer, the signals of interactions mediated by

271 the gear teeth of each intervening subunit are allosterically propagated to the

272 active sites, which reduce their conformational dynamics and in turn, unlock the

273 catalytic potential of GSII (Fig. 4e).

\section{Activation of the GSIl by the 14-3-3 scaffold protein}

275 Having mechanistically established the allosteric coupling between GSIl activity and its

276 quaternary assembly status, we next asked whether there exist cellular factors that may

277 regulate the CsGSIb activity, potentially via favoring its decamer assembly. 14-3-3

278 proteins are an important family of scaffold proteins that bind and regulate many key 
279 proteins involved in diverse intracellular processes in all eukaryotic organisms ${ }^{18-20}$. In 280 particular, self-dimerization of 14-3-3 proteins, which induces dimerization of their

281 clients, plays a key role in its functional scaffolding and subsequent activity regulation $28218,19,21$. Moreover, it has been reported that 14-3-3 proteins act as an activator of GSs in 283 various plants ${ }^{22-25}$, although the detailed activation mechanism remains unclear. Based 284 on these findings, here we tentatively provide the missing link in mechanistically 285 assigning the role 14-3-3 proteins play in regulating GS activity: One protomer of the 14286 3-3 protein recognizes one phosphorylated GSII pentamer, and its self-dimerization 287 brings two pentamer rings in close proximity and therefore promotes decamer assembly, 288 which, in turn, switches on the GS activity via allosteric rigidification of the catalytic sites 289 (Fig. 5a). One prerequisite for this proposal is that, for the GS species whose activities 290 being 14-3-3 protein-dependent, they must have an intrinsically weak decamer-forming 291 propensity that is to be overcome by 14-3-3. In support of this, the GS from Medicago 292 truncatula, whose activity is simulated upon binding to $14-3-3$ protein ${ }^{24}$, has been 293 shown to exhibit a dynamic pentamer-decamer transition ${ }^{12}$, similar to the CsGSIb 294 presented here (Fig. 1d). Moreover, it has been shown that only the higher order 295 complex of tobacco GS-2 that is bound to $14-3-3$ is catalytically active ${ }^{25}$.

296 To further support the above proposal, we then explored whether the activity of the 297 weak-decamer forming CsGSIb could also be regulated by 14-3-3 scaffold protein. 298 Homology search against tea plant genome revealed several candidate tea plant Cs14299 3-3 proteins (Fig. S11). Analysis of their coding genes' expression patterns in tea plant 300 tissues and in nitrogen assimilation or metabolism-related processes allowed us to 301 identify Cs14-3-3-1a and Cs14-3-3-1b genes that displayed expression patterns highly 
302 similar to CsGSI genes (Fig. 5a and S12). Moreover, the expression levels of Cs14-3-

303 3-1a and Cs14-3-3-1b genes were regulated upon changes in the availability of

304 ammonia (Fig. 5b and 5c), the substrate of GS, suggesting both Cs14-3-3-1a and Cs14-

$3053-3-1 b$ are physiologically related to GS. We then examined the in vivo interactions

306 between Cs14-3-3-1a and CsGSIb using the bimolecular fluorescence complementation

307 (BiFC) technique, which is based on complementation between two non-fluorescent

308 fragments of a fluorescent protein when they are brought together by interactions

309 between proteins fused to each fragment ${ }^{26}$. Cs14-3-3-1a or CsGSlb were fused in

310 frame with $\mathrm{N}$-terminal half of a yellow florescence protein (NYFP) or C-terminal half of a

311 yellow florescence protein (CYFP), respectively, and expressed in tobacco leaf

312 epidermal cells alone or in various combinations, such as CsGSIb ${ }^{\text {CYFP }}$ alone or together

313 with Cs14-3-3-1 $\mathrm{a}^{\mathrm{NYFP}}$. As expected, Cs14-3-3-1a and $1 \mathrm{~b}$ could self-dimerize or form

314 heterodimers in plant cells (Fig. 5e), consistent with 14-3-3 scaffold proteins adopting a

315 dimeric structure ${ }^{18,19,21}$. Importantly, formation of the fluorescent complex clearly

316 demonstrated the interaction of Cs14-3-3-1a with CsGSIb (Fig. 5f). In order to further

317 establish the functional relevance, we performed the RNA interference (RNAi) technique

318 to knock down the transcript level of Cs14-3-3-1a gene in hairy roots of chimerical

319 transgenic tea seedlings (Fig. 5h), and evaluated the impact on GS activity by

320 measuring the contents of GS catalysis product, glutamine. We show that, along with

321 the reduction in Cs14-3-3-1a transcript level, the glutamine contents (Fig. 5i), as well as

322 the crude enzyme activity (Fig. 5j), were drastically reduced, indicating the 14-3-3

323 protein in Camellia sinensis functions as an activator molecule of CsGSIb. Further work 
324 is needed to elucidate the detailed mechanism of how Cs14-3-3-1a recognizes

325 phosphorylated CsGSIb.

\section{Discussion}

328 Proper assembly of individual protein units into functional complexes is fundamental to

329 nearly all biological processes. Comparing to the oligomer assembled in relatively 330 simple cyclic symmetry that contains only interfaces of subunits related by the rotational 331 symmetry, protein complexes organized in dihedral symmetry, an extra step of 332 assembly during evolution, possess interfaces that are related by both the rotational 333 symmetry and the perpendicular two-fold axes. However, in many cases, the functional

334 demand for such structural complexity remains poorly understood. Here, by using GS 335 as a model system, we unveil a previously uncharacterized allosteric code buried in a 336 supramolecular protein complex with dihedral symmetry, and show how dynamic 337 packing of protein subcomplexes could build an extra allosteric control for activity 338 modulation.

\section{Dynamics-driven allostery induced by assembly status transition}

340 Allostery describes the mechanism that binding effector molecules at one site triggers

341 a conformational or dynamic change at a distant site, thereby affecting protein activity.

342 Allosteric regulation is a common mechanism to regulate protein function, playing 343 critical roles in various cellular activities ranging from the control of metabolic 344 mechanisms to signal-transduction pathways ${ }^{27}$. Based on the data from Allosteric 345 Database ${ }^{28}$, to date more than 1,900 proteins have been defined as allosteric. Most 346 allosteric modulators identified are small ligands or peptides, whereas in some rare 
347 cases the allosteric effects are induced by protein oligomerization in a rather simple

348 system ${ }^{29}$. Here we show that the oligomeric GS ring functions as positive modulator,

349 the largest allosteric modulator identified so far to our knowledge; and the ring-ring

350 association, which is motivated by 14-3-3 protein or other factors, leads to a transition of

351 assembly symmetry from C5 to D5 and subsequently triggers allosteric activation.

352 We show here that the assembly geometry of GS plays a critical role in determining

353 the protein functional motion properties (Fig. 4). Indeed, protein internal dynamics have

354 been shown essential for functions; and allosteric proteins can be regulated

355 predominantly by changes in their structural dynamics ${ }^{30-33}$. The dynamics-driven

356 allosteric mechanism presented here, in which the GSIl activity is allosterically regulated

357 by the change in conformational fluctuating properties of active sites via inter-ring

358 communication, provides another fascinating example of the interplay between a

359 protein's dynamics and function.

360 The allosteric mechanism of GSIl offers a robust and tunable regulatory 361 machinery.

362 Being a key enzyme implicated in many aspects of the complex matrix of nitrogen

363 metabolism, GS must be strictly regulated. Decades of efforts have been applied to

364 understand how GS is controlled at gene, transcript and protein levels ${ }^{34}$. Studies have

365 demonstrated positive cooperativity of GS with regard to different substrates and

366 cofactors, such as L-glutamate ${ }^{35}$ and metal cations ${ }^{36}$; and GS functions are regulated

367 by multiple post-translational mechanisms including nitration, oxidative turnover and

368 phosphorylation ${ }^{37}$, and by the $14-3-3$ protein ${ }^{22-25}$. Our results reconcile with many of

369 the above observations, and allow us to gain a more complete picture of how GS 
370 activity is regulated in cells by an exquisite machinery (Fig. 6). While the protein

371 turnovers machineries to adjust cellular enzyme level can always provide means to

372 modulate pentamer-decamer transitions and thus deactivation-activation conversion of

373 GSII, we argue that phosphorylation-dephosphorylation processes, coupled with 14-3-3

374 binding and subsequent allosteric activation, may enable a more efficient regulatory way.

375 When sufficient reaction products are available demanding low glutamine synthesis

376 activity, GSII is kept in the dephosphorylated state by certain phosphatase and exists as

377 isolated inactive single-ringed pentamers. In the physiological context of high demand of

378 glutamine, phosphorylation of GSIl by certain kinase prompts 14-3-3 protein binding,

379 and the intrinsic dimerization property of 14-3-3 recruits two GSIl pentamer rings in

380 close proximity and in doing so, result in a rapid transition of quaternary assembly from

381 the pentamer to decamer, and eventually enzymatic activation. In this manner, the

382 poised GSII pentamer ring itself acts as a positive effector and the allosteric ring-ring

383 association offers a great advantage of immediate response to precisely meet the ever-

384 changing metabolic needs, whereas the reversible assembly-disassembly behavior

385 enables a tunable mode for activity modulation. Indeed, the dynamic association-

386 disassociation of GSII subcomplexes, a prerequisite for this modulatory machinery,

387 have been widely observed in various species including humans ${ }^{38}$, plants other than

388 Camellia sinensis reported here ${ }^{12,15}$ and fungi ${ }^{39}$. Therefore, the dynamics-driven

389 allostery shown here may represent a general regulatory machinery harnessed by many

390 eukaryotes to ensure optimal utilization of nitrogen sources, and the infrastructure of

391 fragile ring-ring contacts evolutionarily chosen by many eukaryotes offers a convenient

392 and robust avenue for activity regulation. 


\section{$393 \quad$ Practical implications}

394 As a crucial enzyme to all living organisms, which is involved in all aspects of nitrogen

395 metabolism, GS has emerged as an attractive target for drug design ${ }^{40}$ and herbicidal

396 compounds development, as well as a suitable intervention point for the improvement of

397 crop yields ${ }^{41}$. However, because the overall geometry of the active site is the most

398 conserved structural element amongst GS enzymes ${ }^{4,7,11}$, the traditional strategy of 399 selective inhibition, which relies heavily on the subtle difference in the active sites from

400 different species, has only achieved limited success. Thus, the regulatory mechanism

401 discovered here will help guide the search for specific inhibitors of potential therapeutic

402 interest. For example, inhibition of the GS in Mycobacterium tuberculosis has long been

403 recognized as a novel antibiotic strategy to treat tuberculosis ${ }^{42-44}$. Our result opens new

404 possibilities to develop chemicals to target the drugable ring-ring interface region and

405 specifically interrupt the interactions between two GS subcomplexes in pathogens or

406 unwanted plants to develop new types of herbicide. Moreover, although overexpression

407 of GS has been investigated extensively for decades with the goal of improving crop 408 nitrogen use efficiency, the outcome has not been consistent ${ }^{41}$. The modulatory "hot409 spots" identified here, which mediate inter-ring communication and in turn stimulate GS 410 activity, will guide engineering catalytically more powerful GSs for crops in which 411 pentamer units only weakly associate, and thus increase plant nitrogen use efficiency 412 and crop production. 


\section{Methods:}

430 Cloning, expression, and protein purification of GSs

431 The target genes encoding CsGSIb from Camellia sinensis (Genbank accession No. MK716208)

432 and GmGS 32 from Glycine $\max$ (Genbank accession No. NM001255403) were cloned into the pET-

433 16b vector (Novagen) containing a $\mathrm{His}_{6}$-tagged-MBP tag followed by a tobacco etch virus (TEV)

434 protease cleavage site at the N-terminus. All constructs were transformed into E. coli Rosetta (DE3)

435 cells, which were cultured in Luria-Bertani (LB) medium at $37^{\circ} \mathrm{C}$ supplemented with ampicillin (100 $436 \mu \mathrm{g} / \mathrm{ml})$ and chloramphenicol $(35 \mu \mathrm{g} / \mathrm{ml})$ to an OD600 $\sim .8$. Cells were induced by the addition of 437 isopropyl- $\beta$-D-1- thiogalactopyranoside (IPTG) to the concentration of $0.3 \mathrm{mM}$, and incubated for 438 additional 16 hours at $18{ }^{\circ} \mathrm{C}$. Cells were harvested by centrifugation at $5000 \mathrm{~g}$ for 20 min and 439 resuspended in lysis buffer $(50 \mathrm{mM}$ Tris- $\mathrm{HCl}, 500 \mathrm{mM} \mathrm{NaCl}, \mathrm{pH} 8$ and $1 \mathrm{mM}$ PMSF). 
440 Cells were subjected to a high-pressure homogenizer, named JN-Mini Pro Low-temperature Ultra-

441 high-pressure cell disrupter (JNBIO) and then centrifuged at $50,000 \mathrm{~g}$ for $30 \mathrm{~min}$ at $4^{\circ} \mathrm{C}$. Proteins

442 were initially purified using Ni Sepharose 6 Fast Flow resin (GE Healthcare). The protein tags were

443 cleaved with His-tagged TEV-protease overnight at $4{ }^{\circ} \mathrm{C}$ while dialyzing against TEV cleavage buffer

444 (50 mM Tris- $\mathrm{HCl}, 100 \mathrm{mM} \mathrm{NaCl}, 1 \mathrm{mM} \beta$-mercaptoethanol, $\mathrm{pH}$ 8). Cleaved sample was collected

445 and run over Ni-NTA column to remove His-tagged TEV and protein tags. Flow-through was

446 collected, concentrated and passed over Hiload 16/600 Superdex 200 column (GE Healthcare) in

$44750 \mathrm{mM}$ Tris- $\mathrm{HCl}, \mathrm{pH} 7.4,100 \mathrm{mM} \mathrm{NaCl}, 0.5 \mathrm{mM} \mathrm{MgCl}_{2}$ and $1.5 \mathrm{mM} \beta$-mercaptoethanol.

$448 \quad$ Multi-angle light scattering (MALS) characterization

449 MALS was measured using a DAWN HELEOS-II system (Wyatt Technology Corporation)

450 downstream of a GE liquid chromatography system connected to a Superdex 200 10/300 GL (GE

451 Healthcare) gel filtration column. The running buffer for the protein samples contained $50 \mathrm{mM} \mathrm{KPi}$

$452(\mathrm{pH} 7.0), 100 \mathrm{mM} \mathrm{NaCl}, 1 \mathrm{mM} \beta$-mercaptoethanol and $0.05 \% \mathrm{NaN}_{3}$. The flow rate was set to $0.5 \mathrm{~mL}$

$453 \mathrm{~min}^{-1}$ with an injection volume of $200 \mu \mathrm{L}$, and the light scattering signal was collected at room

454 temperature $\left(\sim 23^{\circ} \mathrm{C}\right)$. The data were analyzed with ASTRA version 6.0.5 (Wyatt Technology

455 Corporation).

456 Glutamine synthetase activity assay

457 GS activity assay was performed described previously ${ }^{45,46}$ and reactions were performed for 30 $458 \min$ at $37^{\circ} \mathrm{C}$ in $50 \mathrm{mM}$ Tris- $\mathrm{HCl}, \mathrm{pH} 7.4,100 \mathrm{mM} \mathrm{NaCl}, 0.5 \mathrm{mM} \mathrm{MgCl}_{2}$ and $1.5 \mathrm{mM} \beta$-mercaptoethanol. 459 Enzymatic activity comparison was conducted with $1 \mu \mathrm{M}$ (monomer) enzyme in the presence of 0.5 $460 \mathrm{mM} \mathrm{NH}_{4} \mathrm{HCl}, 2 \mathrm{mM} \mathrm{L-glutamate,} 0.5 \mathrm{mM} \mathrm{ATP}$. Steady-state kinetic analysis was performed under 461 the same conditions except with the variable concentration of ammonium chloride from $0.05 \mathrm{mM}$ to 4 $462 \mathrm{mM}$. Steady-state kinetic parameters were determined by double reciprocal Lineweaver-Burk plot for 463 reactions that followed Michaelis-Menten kinetics. All experiments were repeated independently at 464 least three times.

$465 \quad$ Fluorescent dye-monitored thermal shift assays 
466 Reactants containing 2uM CsGSIb (monomer) and 1000-fold diluted Sypro Orange in 50mM Tris-

$467 \mathrm{HCl}, \mathrm{pH} 7.4,100 \mathrm{mM} \mathrm{NaCl}$ and $1.5 \mathrm{mM} \beta$-mercaptoethanol were performed using an iCycler 468 thermocycler (Bio-Rad) as previous described ${ }^{13}$. Briefly, CsGSIb in presence of various 469 concentrations of the following ligands were tested, alone and in combination: 10mM glutamate, $47020 \mathrm{mM} \mathrm{MgCl}_{2}$ and $1 \mathrm{mM} \mathrm{ATP}$. The temperature of the reactions was increased from 20 to $90{ }^{\circ} \mathrm{C}$ in 471 increments of $0.2{ }^{\circ} \mathrm{C} / 12 \mathrm{~s}$, coincident with a fluorescent measurement at each step. The 472 wavelengths for excitation and emission were set to 490 and $575 \mathrm{~nm}$, respectively. Fluorescence 473 changes were monitored simultaneously with a charge-coupled device (CCD) camera. To obtain the 474 temperature midpoint for the protein unfolding transition, Tm, a Boltzmann model was used to fit the 475 fluorescence imaging data obtained by the CCD detector using the curve-fitting software GraphPad 476 Prism 7.0.

\section{$477 \quad$ Analytical ultracentrifugation (AUC)}

478 Sedimentation velocity experiments were carried out with a Proteomelab XL-A analytical 479 ultracentrifuge (Beckman Coulter, USA) using a four-hole An-60 Ti analytical rotor. An aliquot of 410 $480 \mu \mathrm{L}$ of buffer $(50 \mathrm{mM}$ Tris- $\mathrm{HCl}, \mathrm{pH} 7.4,100 \mathrm{mM} \mathrm{NaCl}$ and $1.5 \mathrm{mM} \beta$-mercaptoethanol) as the 481 reference and $400 \mu \mathrm{L}$ of protein solution $\left(0.1 / 0.25 / 0.5 \mathrm{mg} \cdot \mathrm{mL}^{-1}\right)$ were loaded into a double-sector 482 cell. A centerpiece with a path length of $12 \mathrm{~mm}$ was used. The speed of rotor was $35,000 \mathrm{rpm}$. The 483 operation temperature of rotor was $20^{\circ} \mathrm{C}$. The time dependence of the absorbance at different radial 484 positions was monitored at a wavelength of $280 \mathrm{~nm}$ by an UV-Vis absorbance detector, and the 485 data were analyzed by the software SEDFIT (version 15.01b) using c(s) model to obtain the 486 sedimentation coefficient distribution. Viscosity and density of the buffer solution were calculated by 487 the Sednterp software.

488 Single-particle cryo-electron microscopy data collection

489 Purified protein samples of CsGSlb (4 $\mu \mathrm{L}, 0.02 \mathrm{mg} / \mathrm{mL})$ and $\mathrm{GmGS} \beta 2(4 \mu \mathrm{L}, 0.02 \mathrm{mg} / \mathrm{mL})$ in 50 $490 \mathrm{mM}$ Tris- $\mathrm{HCl}, \mathrm{pH} 7.4,100 \mathrm{mM} \mathrm{NaCl}$, and $1.5 \mathrm{mM} \beta$-mercaptoethanol were negatively stained with 491 uranyl acetate $1 \%(w / v)$ on carbon-film 400 mesh copper grids. Samples were imaged using a FEl 492 T12 operated at $120 \mathrm{keV}$ with a $3.236 \AA$ pixel size, $68,000 \times$ nominal magnification, and defocus 
493 range about $1.5 \mu \mathrm{m}$. For cryo-EM, $3 \mu \mathrm{L}$ of CsGSlb $(0.1 \mathrm{mg} / \mathrm{mL}$ and $0.5 \mathrm{mg} / \mathrm{mL})$ and $\mathrm{GmGS} \beta 2(0.1$

$494 \mathrm{mg} / \mathrm{mL}$ ) were added onto glow-discharged Quantifoil R1.2/1.3 100 holey-carbon Cu grids with a

495 Vitrobot Mark IV (Thermo Fisher Scientific). The grids were blotted for $3.5 \mathrm{~s}$ at $8{ }^{\circ} \mathrm{C}$ with $100 \%$

496 humidity, and then plunged frozen into liquid ethane cooled by liquid nitrogen. Cryo-grids were first

497 screened on a FEI TF20 operated at $200 \mathrm{keV}$. Images of CsGSIb and GmGS $\beta 2$ were collected using

498 Titan Krios G3i microscope (FEl) operated at 300 kV with a Gatan K2 Summit direct detection

499 camera. Two datasets were acquired using the SerialEM in super-resolution mode with a nominal

500 magnification of $29,000 x$, yielding a pixel sizes of $0.505 \AA$ with a total dose of $51 \mathrm{e} / \AA^{2}$. The defocus

501 ranges were set from $-1.6 \mu \mathrm{m}$ to $-2.3 \mu \mathrm{m}$.

502 Cryo-electron microscopy image processing, 3D reconstruction, and analysis

503 All processing steps were performed using cryoSPARC ${ }^{47}$. A total of 4,051 raw movie stacks

504 acquired for CsGSIb and 1,777 raw movie stacks for GmGS $\beta 2$ were subjected to patch motion

505 correction and patch CTF estimation. An initial set of about 500 particles were manually picked to

506 generate 2D templates for auto-picking. The auto-picked particles were extracted by a box size of

507512 pixel and then subjected to reference-free 2D classification. After particle screening using 2D

508 and 3D classification, the final 355,289 particles for CsGSIb and 115,795 particles for GmGS $\beta 2$ were

509 subjected to Ab-Initio Reconstitution and followed by 3D Refinement with C5 symmetry imposed.

510 Four different conformational states were obtained for CsGSIb, resulting in a $3.3 \AA$ density map for

$511 \mathrm{CsGSIb}^{\mathrm{Dec}}, 3.5 \AA$ density map for CsGSIb ${ }^{\text {Pen }}$ State I, $3.6 \AA$ density map for CsGSIb ${ }^{\text {Pen }}$ State II, and

$5123.4 \AA$ density map for CsGSIb ${ }^{\text {Pen }}$ State III. Only one major conformation was obtained with $2.9 \AA$

513 density map for GmGSß2. The global resolution of the map was estimated based on the gold-

514 standard Fourier shell correlation (FSC) using the 0.143 criterion.

$515 \quad$ Model building and structural refinement

516 Homology models of CsGSIb and GmGSß2 were generated with the I-TASSER server ${ }^{48}$ and 517 docked into the cryoEM maps using UCSF Chimera ${ }^{49}$. The sequences were mutated with 518 corresponding residues in CsGSIb and GmGSß2, followed by rebuilding in Coot ${ }^{50}$. The missing 519 residues of $\mathrm{CsGSIb}^{\text {Pen }}$ were not built due to the lack of corresponding densities. Real-space 
520 refinement of models with geometry and secondary structure restraints applied was performed using 521 PHENIX ${ }^{51}$. The final model was subjected to refinement and validation in PHENIX. The statistics of 522 cryo-EM data collection, refinement and model validation are summarized in Table S1.

523 Different nitrogen treatments for hydroponically grown tea cuttage seedlings

524 Two-year-old hydroponic tea cuttage seedlings were grown in a greenhouse at $20-25^{\circ} \mathrm{C}$ until new 525 tender roots emerged. These healthy tea seedlings were then transferred into hydroponic solutions 526 with different nitrogen sources, namely, $0 \mathrm{mM} \mathrm{NH}_{4}{ }^{+}$(Shigeki Konishi solution), $5 \mathrm{mM} \mathrm{NH}_{4}{ }^{+}$(Shigeki 527 Konishi solution with $5 \mathrm{mM}$ ammonium nitrogen), $10 \mathrm{mM} \mathrm{NH}_{4}^{+}$(Shigeki Konishi solution with $10 \mathrm{mM}$ 528 ammonium nitrogen), and a control (Shigeki Konishi solution alone). All of these tea seedling root 529 samples were cleaned and collected in liquid nitrogen after treatment for RNA analysis.

$530 \quad$ RNA isolation and qRT-PCR analysis

531 Tea plant tissues or root materials were ground in liquid nitrogen into fine powders for total RNA 532 extraction with an RNA extraction kit (Tiangen Biotech Co., Ltd.) according to the manufacturer's 533 instructions. RNA quality and purity were assessed by a NanoDrop 2000 spectrophotometer 534 (Thermo Scientific). The integrity of the RNA samples was rapidly checked by $1.0 \%$ agarose gel 535 electrophoresis. The total RNA was reverse-transcribed to single-stranded cDNAs using SuperScript 536 III reverse transcriptase (Invitrogen) according to the manufacturer's instructions. qRT-PCR analysis 537 was performed using cDNA synthesized by the Prime Script RT Reagent Kit (TaKaRa). Each qRT538 PCR was conducted in a $20-\mu \mathrm{L}$ reaction mixture containing $2 \mu \mathrm{L}$ of diluted template cDNA, $0.4 \mu \mathrm{L}$ of 539 each specific primer, $10 \mu \mathrm{L}$ of SYBR Premix Ex-Taq (TaKaRa), and $7.2 \mu \mathrm{L}$ of $\mathrm{H}_{2} \mathrm{O}$. All qRT-PCR 540 assays were performed on the Bio-Rad CFX96 fluorescence-based quantitative PCR platform. The 541 program used was as follows: $95^{\circ} \mathrm{C}$ for 5 minutes; 40 cycles of $95^{\circ} \mathrm{C}$ for $5 \mathrm{~s}$ for denaturation and $54260^{\circ} \mathrm{C}$ for $30 \mathrm{~s}$ for annealing and extension; and 61 cycles of $65^{\circ} \mathrm{C}$ for $10 \mathrm{~s}$ for melting curve analysis. 543 All experiments were independently repeated three times, and relative expression levels were 544 measured using the $2-\Delta$ Ct method.

545 Subcellular localization of CsGSIs and Cs14-3-3-1a\&1b 
546 Construction of the Cs14-3-3-1a-GFP, CsGSI-1b-GFP, and Cs14-3-3-1b-GFP fusions were

547 performed using gateway recombination systems. The corresponding ORFs for CsGSIb and Cs14-3-

548 3-1a, 1b were subcloned into pK7WGF2 in frame with a GFP tagged at the N-terminus.

549 Determination of the subcellular localization of these GFP fusions was performed using tobacco leaf

550 infiltration as previously described (Zhao et al., 2011). Briefly, the pK7WGF2-Cs14-3-3-1a-GFP,

551 pK7WGF2-Cs14-3-3-1b-GFP, and pK7WGF2-CsGSIb-GFP plasmids were transformed into $A$.

552 tumefaciens strain EHA105, and selected positive colonies harboring these constructs were used for

553 plant transformation by infiltration. Acetosyringone-activated Agrobacterium cells were infiltrated into

554 the Nicotiana benthamiana leaves leaf abaxial epidermal surface, and the tobacco plants were 555 grown at room temperature for 3 days before imaging. Imaging of these GFP fusion proteins was 556 performed using a confocal microscope with a 100x water immersion objective and appropriate 557 software. The excitation wavelength was $488 \mathrm{~nm}$, and emissions were collected at $500 \mathrm{~nm}$.

558 In planta interaction between CsGSlb with Cs14-3-3 proteins with Bimolecular

559 Fluorescent Complimentary (BIFC)

560 The ORFs of Cs14-3-3-1a, Cs14-3-3-1b, and CsGSIb were amplified and subcloned into 561 pCAMBIA1300-eYFPN (YFP N-terminal portion) and pCAMBIA1300-eYFPC (YFP C-terminal portion) 562 (CAMBIA) by the in-fusion technology. The resulting constructs were transformed into $A$. 563 tumefaciens strains GV3101, which were infiltrated into Nicotiana benthamiana leaves individually or 564 in different pair combinations. A Leica DMi8 M laser scanning confocal microscopy system was used 565 for fluorescence observation, according to the method described previously ${ }^{52}$. If the fluorescence 566 signal could be detected with any interaction pair, the pair of half YFP-fusion proteins should interact.

$567 \quad$ Knockdown of Cs14-3-3-1a in tea plant hairy roots

568 Approximately $400 \mathrm{bp}$ of the gene-specific fragments from Cs14-3-3-1a were amplified and 569 subcloned into the final RNA interference (RNAi) destination vector pB7GWIWG by BP and LR 570 clonase-based recombination reactions (Invitrogen). The resulting binary vectors pB7GWIWG-Cs14571 3-3-1a were transformed into A. rhizogenes strain ATCC 15834 by electroporation. The selected 
572 positive transformants harboring pB7GWIWG-Cs14-3-3-1a were used to transform 3-month-old tea

573 seedlings, which were pretreated with acetosyringone. The positive transgenic hairy root lines were

574 verified with qRT-PCR for examination of transgene expression. At least three independent hairy

575 root lines were selected for further analysis.

576 Determination of free amino acids in tea plant samples

577 The free amino acids in tea plant samples were analyzed by using an amino acid analyzer (L-8900,

578 Hitachi) according to manufacture instruction. The free amino acids were extracted from $120 \mathrm{mg}$

579 leaves with $1 \mathrm{~mL}$ of $4 \%$ sulfosalicylic acid in water bath sonication for $30 \mathrm{~min}$ and then centrifuged at

$58013,680 \times \mathrm{g}$ for $30 \mathrm{~min}$. The debris was re-extracted once again and the supernatants from two

581 extractions were combined as previously described ${ }^{53}$. The supernatants were filtered through a

$5820.22 \mu \mathrm{m}$ Millipore filter before analysis. A mobile phase containing lithium citrate for amino acid

583 derivatization and UV-Vis detection at 570 and $440 \mathrm{~nm}$ were used in the Hitachi High-Speed Amino

584 Acid Analyzer system. The flow rates were set at $0.35 \mathrm{~mL} / \mathrm{min}$ for the mobile phase and $0.3 \mathrm{~mL} / \mathrm{min}$

585 for the derivatization reagent. The temperature for separation column was set to $38{ }^{\circ} \mathrm{C}$, and for the

586 post-column reaction equipment was maintained at $130^{\circ} \mathrm{C}$. The temperature of the autosampler was

587 kept at $4{ }^{\circ} \mathrm{C}$. The peak areas of amino acids were quantified in comparison with the amino acid

588 standards.

$589 \quad$ GS activity assay from plant samples

590 To determine the total GS activity, $100 \mathrm{mg}$ of frozen plant samples were grounded into fine powder

591 in liquid nitrogen. Samples were homogenized in extraction buffer $(50 \mathrm{mmol} / \mathrm{L}$ Tris- $\mathrm{HCl}, \mathrm{pH} 8.0$,

$5922 \mathrm{mmol} / \mathrm{L} \mathrm{MgSO}_{4}, 4 \mathrm{mmol} / \mathrm{L}$ dithiothreitol, and $0.4 \mathrm{mmol} / \mathrm{L}$ sucrose). Plant extracts were centrifuged

593 at $13,680 \times \mathrm{g}\left(4^{\circ} \mathrm{C}\right)$ for $25 \mathrm{~min}$ and the supernatants of extracts were analyzed for the soluble protein

594 content using the Bradford assay. GS activity was determined after incubating the enzyme extracts

595 in a reaction buffer $\left(100 \mathrm{mmol} / \mathrm{L}\right.$ Tris- $\mathrm{HCl}, 80 \mathrm{mmol} / \mathrm{L} \mathrm{MgSO}_{4}, 20 \mathrm{mmol} / \mathrm{L}$ sodium glutamate, $80 \mathrm{mM}$

$596 \mathrm{NH}_{4} \mathrm{OH}, 20 \mathrm{mmol} / \mathrm{L}$ cysteine, $2 \mathrm{mmol} / \mathrm{L}$ EGTA and $40 \mathrm{mmol} / \mathrm{L} \mathrm{ATP}$ ) at $37^{\circ} \mathrm{C}$ for $30 \mathrm{~min}{ }^{54}$. A stop

597 solution containing $0.2 \mathrm{~mol} / \mathrm{L}$ Trichloride acetic acid, $0.37 \mathrm{~mol} / \mathrm{L} \mathrm{FeCl}$ and $0.6 \mathrm{~mol} / \mathrm{L} \mathrm{HCl}$ was added; 
bioRxiv preprint doi: https://doi.org/10.1101/2021.08.20.457084; this version posted August 20, 2021. The copyright holder for this preprint (which was not certified by peer review) is the author/funder, who has granted bioRxiv a license to display the preprint in perpetuity. It is made available under aCC-BY 4.0 International license.

598 and the absorbance of enzyme reactions at $540 \mathrm{~nm}$ was recorded. A standard curve was made in an

599 identical way for calculation of the specific enzyme activity.

600

601

602

603

604

605

606

607

608

609 
a

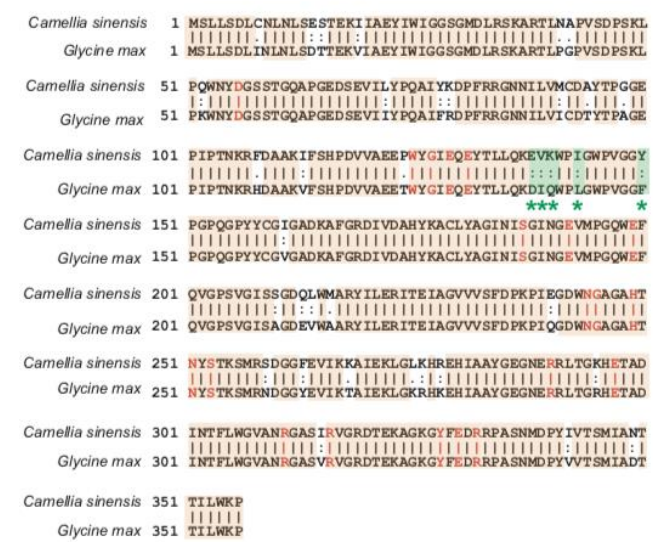

e

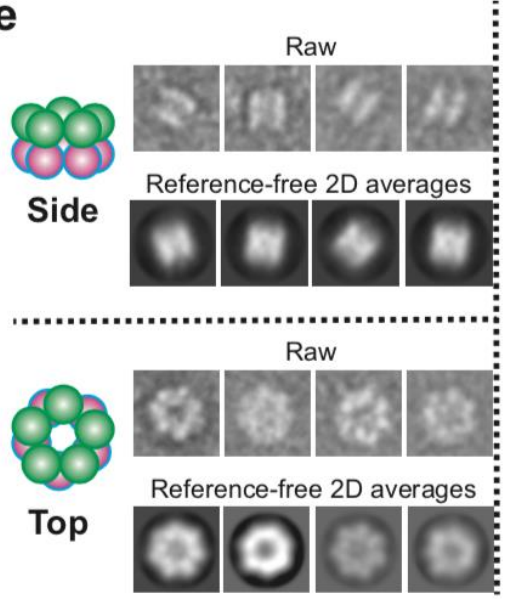

b

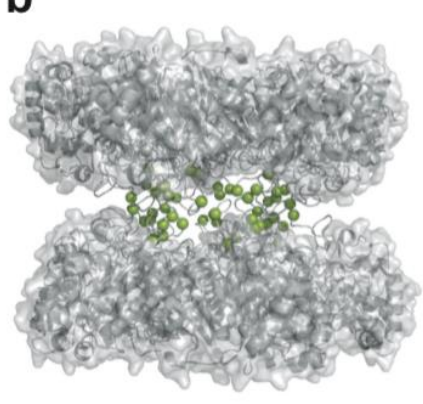

f

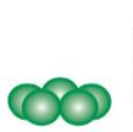

Side

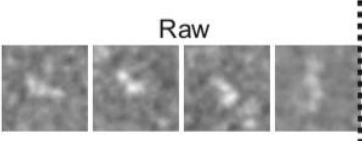

Reference-free 2D averages
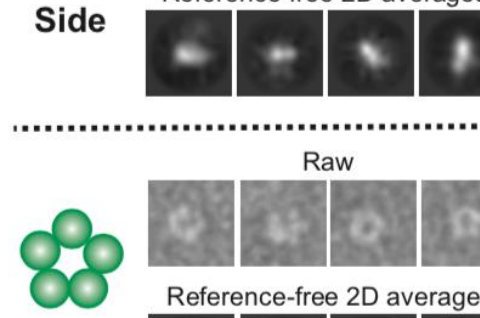

Top
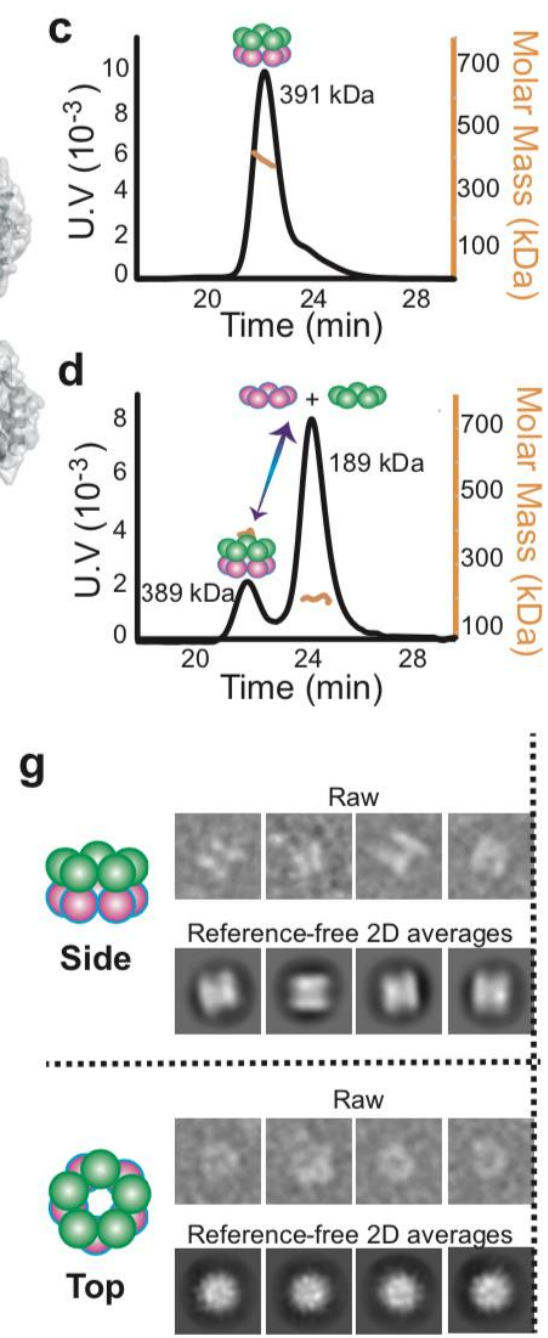

611 Fig. 1: Quaternary assembly property comparison of GSIls from Camellia sinensis

612 (CsGSIb) and Glycine $\max (\mathrm{GmGS} \beta 2)$.

613 a, Amino acid sequence alignment of CsGSIb and GmGS $\beta 2$ reveals very high level of

614 conservation. Identical amino acids are shown with orange boxes, while the residues

615 involved in substrate-binding and catalysis are shown in red. Amino acids variations

616 located at the pentamer ring-ring interface are highlighted in green boxes with the

617 symbol of ${ }^{*}$. b. Model structure built based on the crystal structure of a maize GSII 
618 (GmGS $\beta 2$, pdb code 2D3B). The amino acid variations between CsGSIb and GmGS $\beta$

619 that are located at the ring-ring interface are highlighted as spheres in green. c-d, SEC-

620 MALS analysis of GmGSß2 (c) and CsGSIb (d). e-g, Quaternary assembly analysis of

621 GmGSß2 and CsGSIb using negative-stain electron microscopy. Left: Examples of

622 single raw images; Middle: reference-free two-dimensional class averages. Right: A

623 schematic representation of the averages is shown for clarity. GmGS $\beta 2$ adopts a

624 homogenous double-ringed structure (e), while the CsGSIb demonstrates a mixture of

625 two major classes of particles: isolated pentamer ring (f) and double-ringed structure ( $\mathrm{g}$ ). 

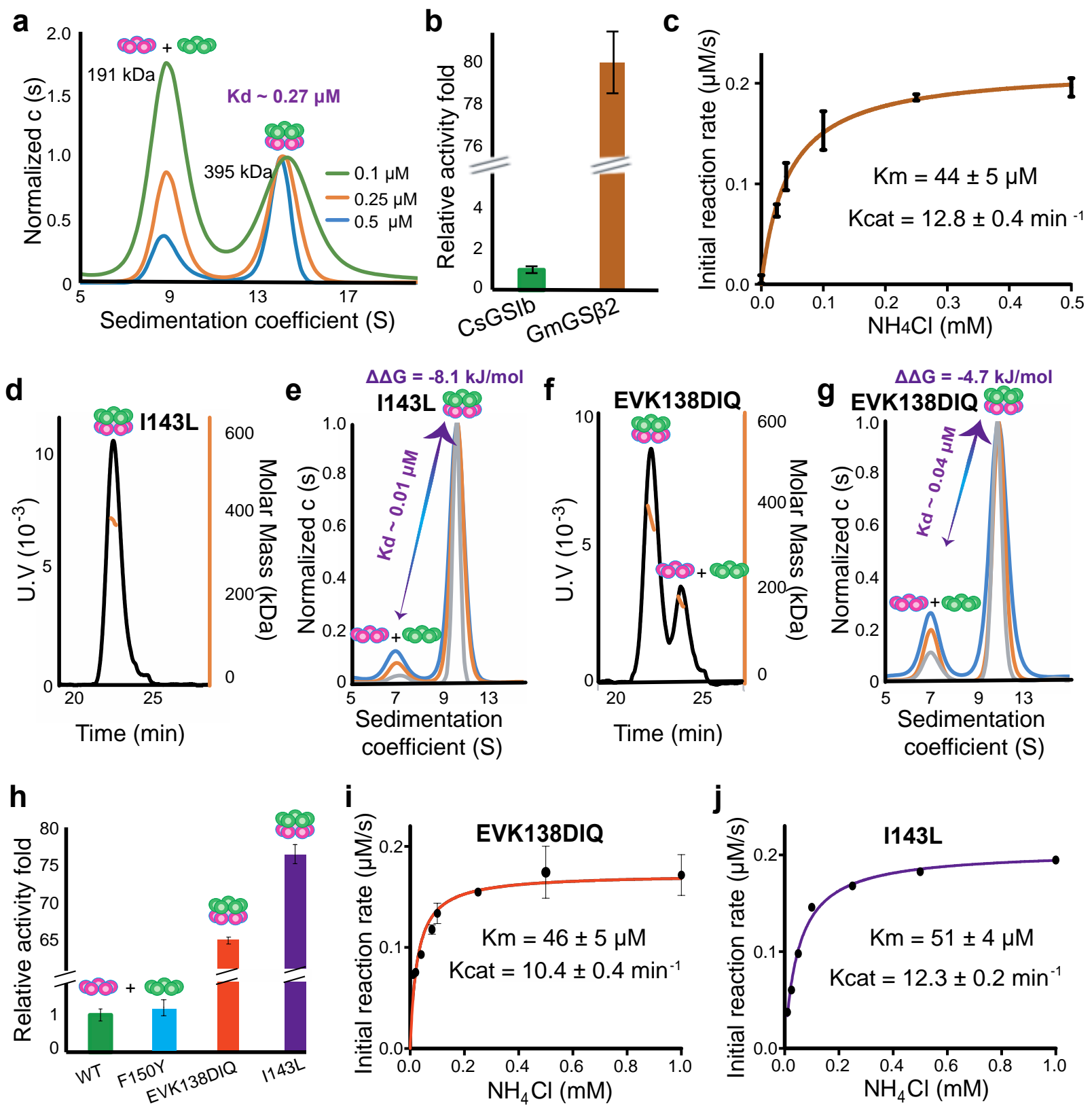

640 Fig. 2: The enzymatic activities of CsGSIb is dependent on its quaternary

641 assembly status.

642 a, Application of analytical ultracentrifugation (AUC) to assess the pentamer-decamer

643 dissociation constant of CsGSIb. Experiments were performed at room temperature with

644 three different sample concentrations shown as monomer concentration. A global fit of 
645 sedimentation distribution profiles yielded a dissociation constant of $0.27 \mu \mathrm{M}$. b,

646 Enzymatic activity comparison of GmGS $\beta 2$ with CsGSIb. Reactions were performed for

$64730 \mathrm{~min}$ at $37^{\circ} \mathrm{C}$ in presence of $1 \mu \mathrm{M}$ (monomer) enzyme and saturated amounts of

648 substrates. c, Steady-state kinetic analysis of GmGS $\beta 2$. Assay conditions were the

649 same as that in $\mathbf{b}$, except the concentrations of $\mathrm{NH}_{4} \mathrm{Cl}$ were varied. $\mathbf{d}-\mathbf{g}$, Mutation effects

650 on the quaternary assembly property of CsGSIb evaluated using SEC-MALS (d and f)

651 and AUC (e and $\mathbf{g}$ ). The corresponding mutants are labeled in the figures. $\mathbf{h}$, Activity

652 comparison of the wild type GmGs18 with its mutants as labeled. Reactions were

653 performed in the same condition as $\mathbf{b}$. $\mathbf{i}-\mathbf{j}$, Steady-state kinetic analysis of GmGS $\beta 2$

654 mutants of EVK138DIQ (i) and I143L (j). Reaction conditions were same as in c. All

655 enzyme assays were repeated at least three times and data were shown as means \pm

656 s.d.

657

658

659

660

661

662

663

664

665

666

667 
a

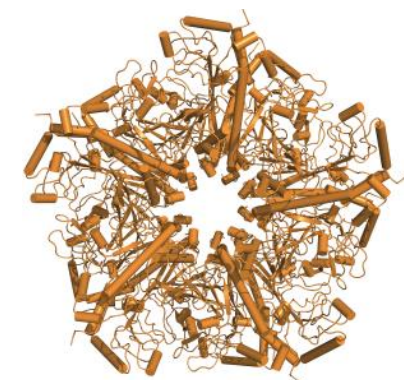

b

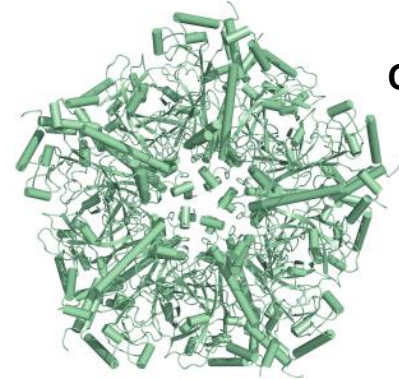

d

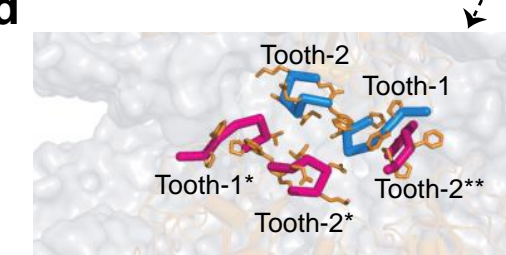

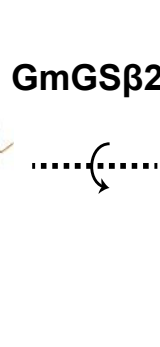

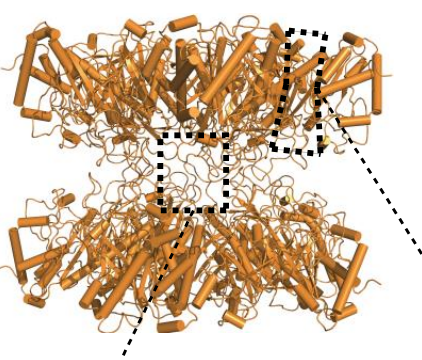

C

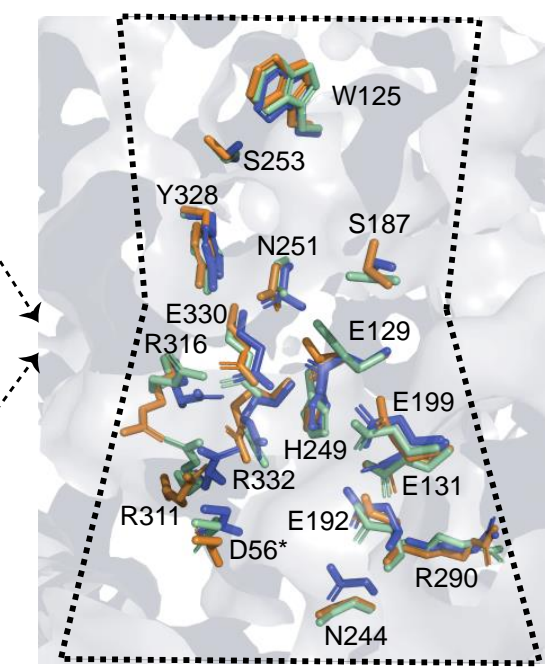

Active site

$\mathbf{f}$

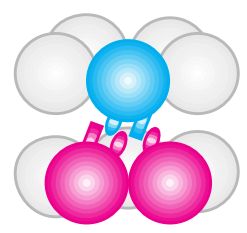

670 Fig. 3: Overall structures, catalytic centers and ring-ring interfaces of GmGS $\beta 2$

671 and CsGSIb ${ }^{\text {Dec }}$.

672 a-b, Overall double-ringed structures of GmGS $\beta$ (a) and CsGSIb ${ }^{\text {Dec }}$ (b). Left: top-view;

673 Right: Side-view. c, Superimposed structures of the catalytic centers of GmGS $\beta 2$

674 (orange), CsGSIb ${ }^{\text {Dec }}$ (green) and GSIl from maize (purple). High structure similarities in

675 catalytic sites suggest the catalytic mechanism for these three GSII species are

676 essentially identical. d-e, The detailed ring-ring interaction interfaces between GmGS $\beta 2$

677 (d) and CsGSIb ${ }^{\text {Dec }}$ (e). The interactions between two pentameric rings are primarily

678 mediated by two regions, namely, the tooth-1 and tooth-2, respectively. (f), A schematic 
680 clarity.

681

b
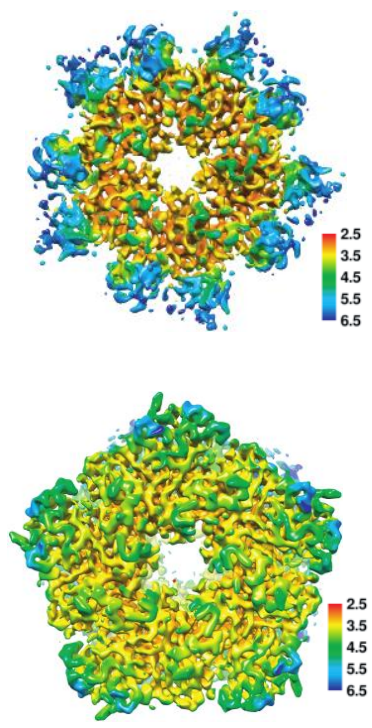
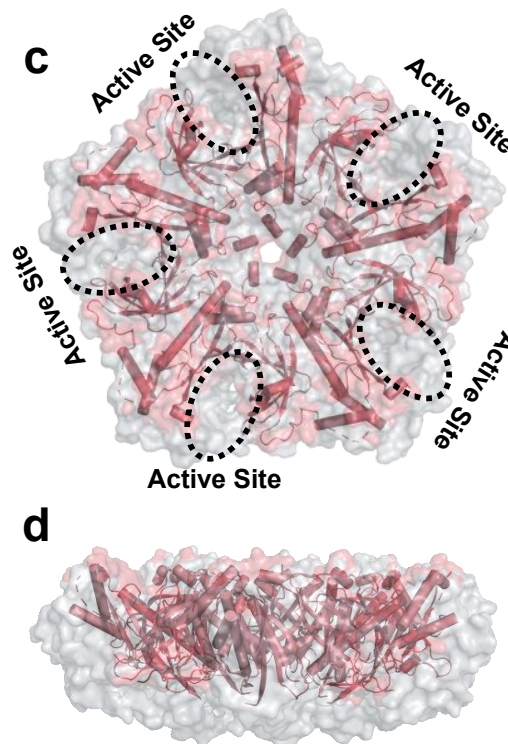

Ring-ring Interface e

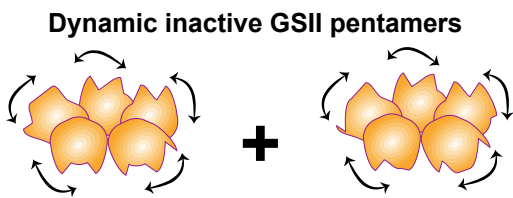

Dynamics-driven
Allosteric
Regulation

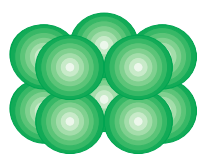

Ragid active GSII decamers

684 three structures are shown in Fig. S9. a, Local resolution of the density map of 685 CsGSIb $^{\text {Pen }}$ indicates a decreased resolution near the edges of the pentamer ring. $\mathbf{b}$, Local resolution of the density map of $\mathrm{CsGSI}^{\mathrm{Dec}}$ map. The conformational flexibility is

687 apparent when the missing density at the rim of $\mathrm{CsGSI}^{\text {Pen }}$, which yields a largely 688 decagram-shaped map (a), is compared to the intact density of CsGSIb ${ }^{\text {Dec }}$ that displays a pentagon-shaped map (b). c-d, Superimposed of Cryo-EM structure of CsGSIb ${ }^{\text {Pen }}$ 690 (pink) with that of CsGSIb ${ }^{\text {Dec }}$ (grey surface). c: Top-view; d: Side-view. The results 691 reveal two major regions being highly disordered: the rim region including the catalytic 692 center, and ring-ring interface. e, Proposed dynamics-driven allosteric model of CsGSIb. 
a

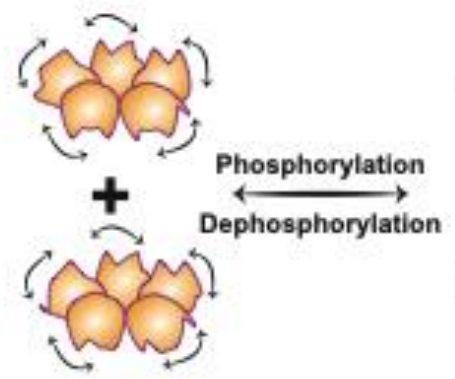

Dynamic inactive GSII pentamers
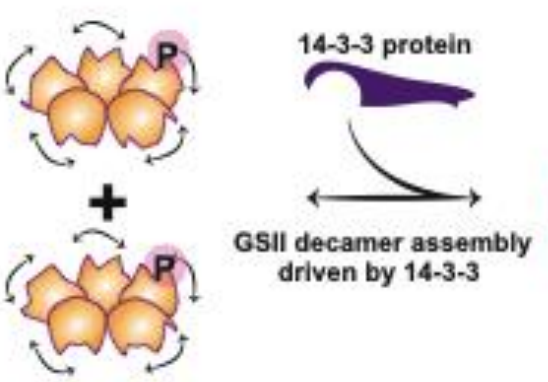

Phosphorylated GSIl pentamers

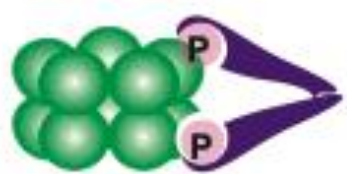

Active GSII decamers activated by 14-3-3

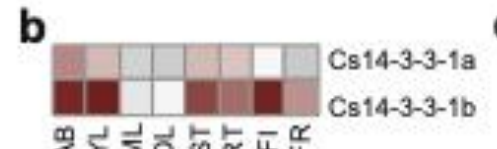

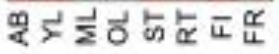

c

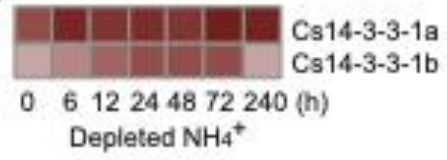

d

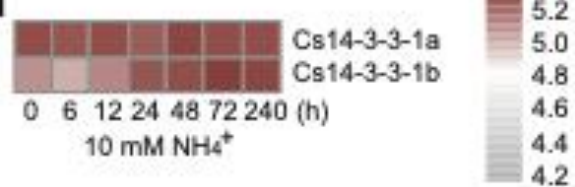

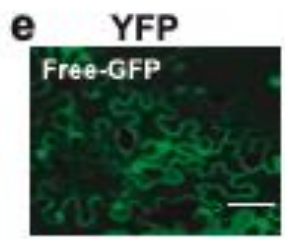
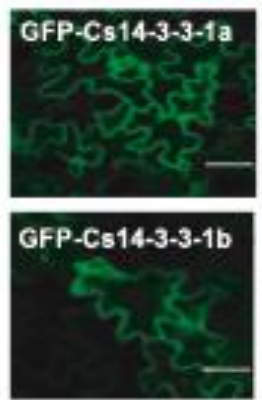

Bright
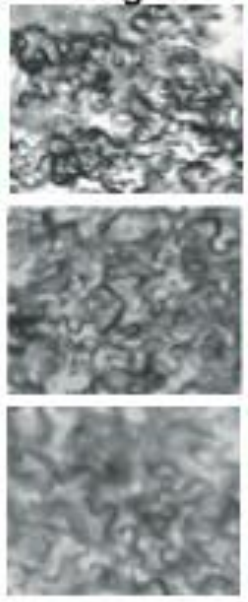
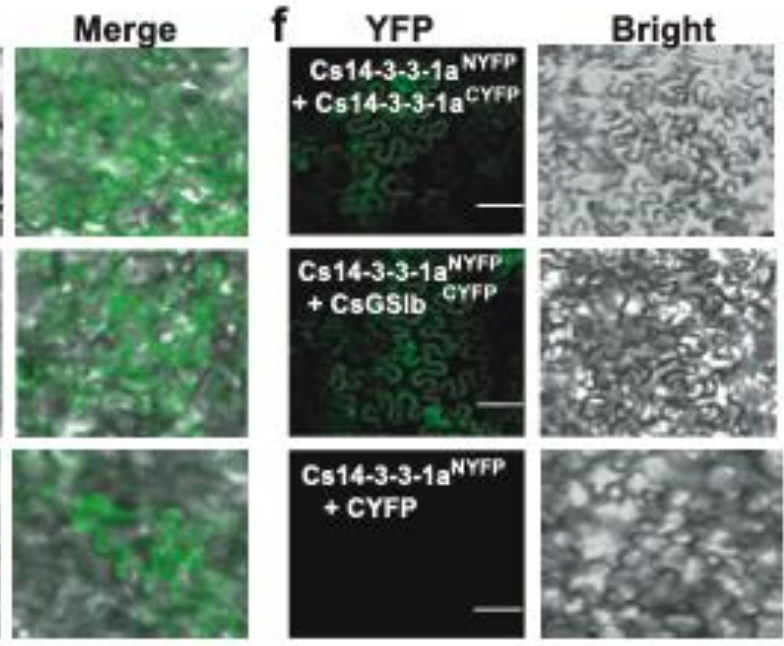

Merge

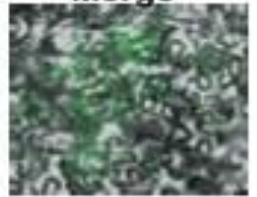

g

h
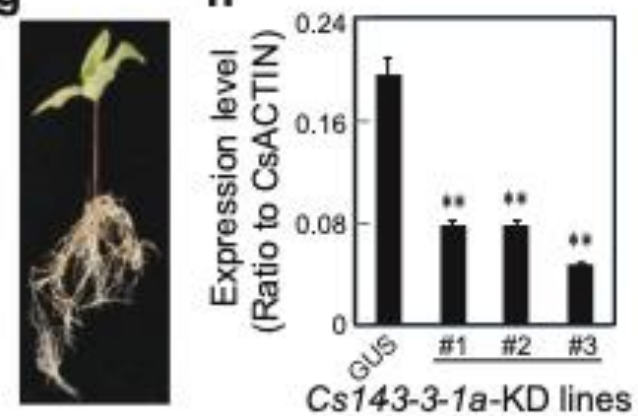

697

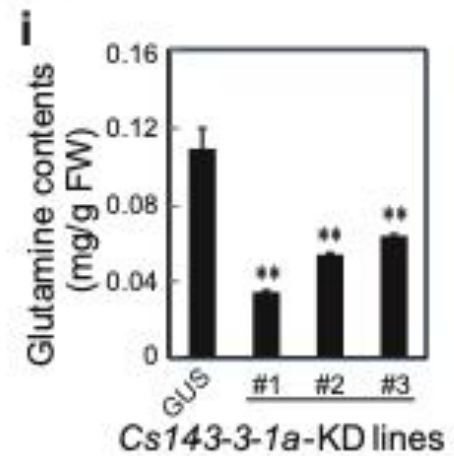

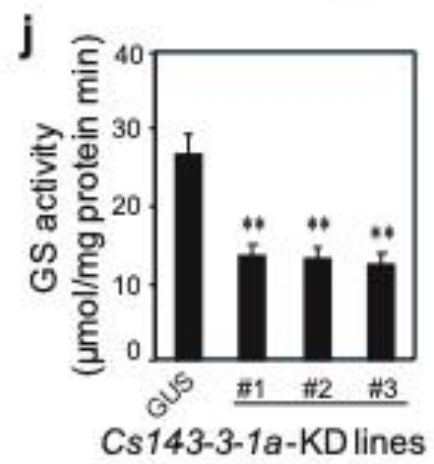

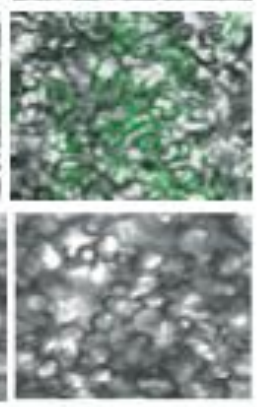


699 a, Proposed working model of how 14-3-3 protein modulates the activity of GSIls. P in

700 pink sphere denotes the post-translational modification of phosphorylation. b,

701 Expression patterns of Cs14-3-3-1a and Cs14-3-3-1b genes in various tea plant tissues.

702 c, Induction of Cs14-3-3-1a and Cs14-3-3-1b genes by depletion of $\mathrm{NH}_{4}{ }^{+}$from culture

703 medium. d, Expression of Cs14-3-3-1a and Cs14-3-3-1b genes in tea plant roots fed

704 with $10 \mathrm{mM} \mathrm{NH}_{4}^{+} . \mathrm{AB}$, apical buds of unopened leaves at the top of actively growing

705 shoots; YL, first and second young leaves below the apical buds; ML, mature leaves

706 geminated in the spring and harvested in the autumn; OL, old leaves at the bottom of

707 tea tree plant; FL, flowers; FR, fruits of tea plants; ST, stem tissues at the 2nd and 3rd

708 internodes; RT, roots. e. Subcellular localization of Cs14-3-3-1a and Cs14-3-3-1a fusion

709 proteins in tobacco leaf epidermal cells. bar $=50 \mu \mathrm{M}$. f. BIFC assay of interaction

710 among Cs14-3-3-1a, Cs14-3-3-1b and CsGSIlb in tobacco leaf cells. bar = $50 \mu \mathrm{M} . \mathbf{g}$

711 Generation of tea plant transgenic hairy roots with RNAi knockdown(KD) of Cs14-3-3-1a

712 gene. $\mathbf{h}$, Expression of Cs14-3-3-1a in at least three tea plant transgenic hairy roots of

713 Cs14-3-3-1a-KD compared with GUS roots. i, Glutamine contents in three tea plant

714 transgenic hairy roots of Cs14-3-3-1a-KD compared with GUS roots. j, GS activity in

715 three tea plant transgenic hairy roots of Cs14-3-3-1a-KD compared with GUS roots. All

716 experiments were conducted at least three 3 independent experiments. At least 5

717 transgenic hairy roots for Cs14-3-3-1a and GUS genes were examined. Data are

718 expressed as means \pm s.d. Differences in two-tailed comparisons between transgenic

719 lines and GUS controls were analyzed, ${ }^{* *} p<0.01$ in student's t-test. See Methods for

720 experimental details. 


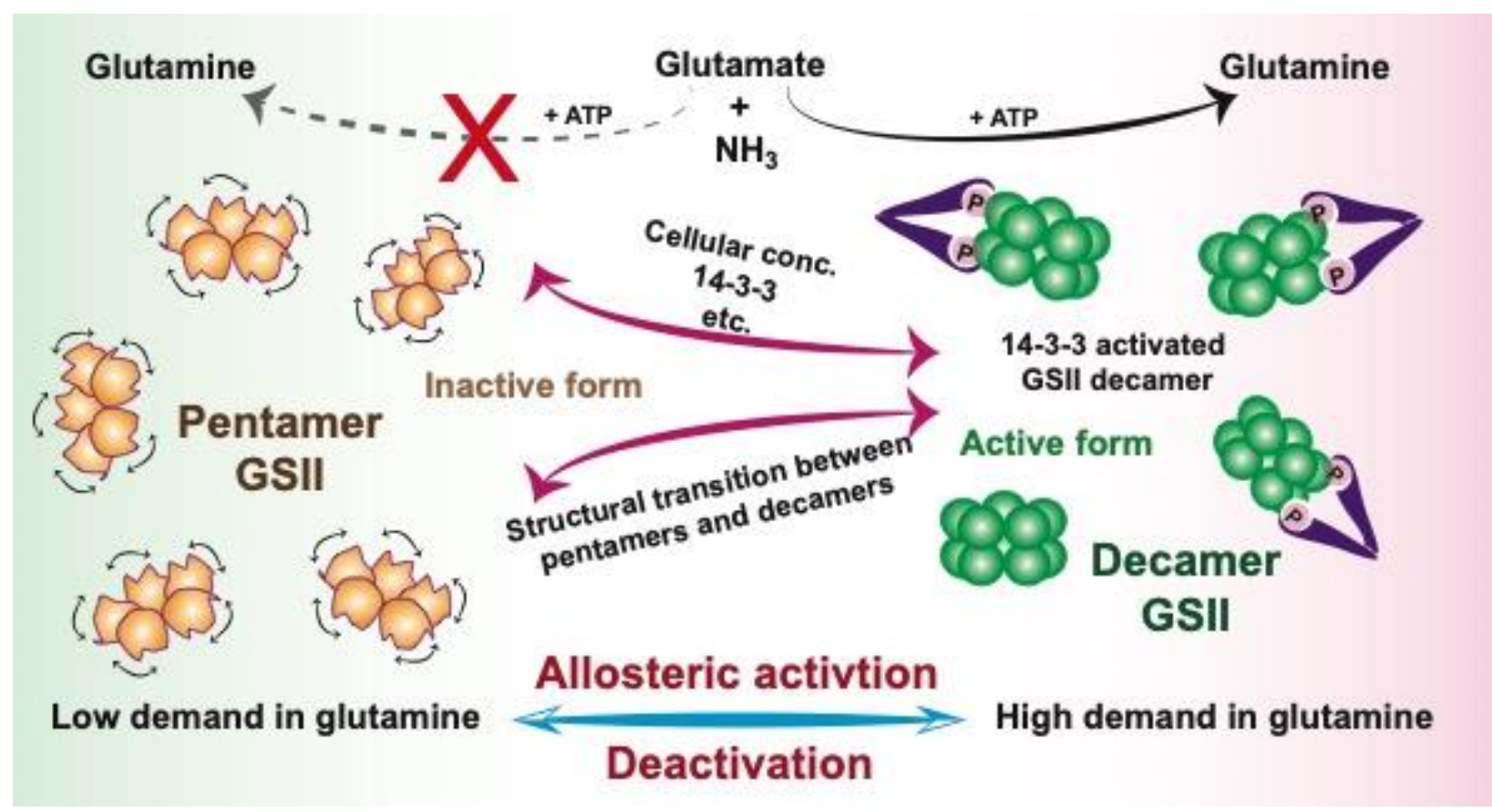

727 Fig. 6: Schematic model of how activity of GSII is allosterically regulated in cells

728 to meet metabolic needs.

729 In low demand of glutamine, GSII is kept in the dephosphorylated state and exists as

730 isolated inactive pentamers (orange); In the physiological context of high demand of

731 glutamine, phosphorylation and subsequent binding to 14-3-3 (or by increase in cellular

732 concentration) trigger a rapid transition of quaternary assembly of GSII from the 733 pentamer to decamer (green), which, via the mechanism of dynamics-driven allostery,

734 activates GSII. 


\section{Supplementary Data \& Figures}

\section{Camellia sinensis CsGSIb exhibits a pentamer-decamer dynamic equilibrium in}

\section{4 solution}

745 To further characterize the low propensity of CsGSIb for decamer forming, we carried

746 out SEC-MALS measurements at various concentrations. As shown in Fig. S1a,

747 increase in the concentration of CsGSIb sample injected favored association of

748 pentamers towards formation of decamer, and vice versa, suggesting a dynamic nature

749 of the interaction between two pentameric rings. Moreover, a series of SEC-MALS

750 measurements of CsGSlb at different salt concentrations were performed to decipher

751 the driving force mediating the inter-ring contact. While addition of salt of $\mathrm{NaCl}$ in the

752 regime of low salt concentrations (from 0 to $300 \mathrm{mM}$ ) provoked the dissociation of

753 decamer into pentamers first, further increase in salt concentration (from 300 to $500 \mathrm{mM}$ )

754 tended to facilitate re-association of pentameric subcomplexes to some extent (Fig.

$755 \mathrm{~S} 1 \mathrm{~b})$, suggesting a mixture of electrostatic and hydrophobic interactions is responsible

756 for attaching of two pentameric rings.

757 To probe the effects of various ligands on the stability of CsGSlb, a series of

758 fluorescent dye-monitored thermal shift assays were carried out as described previously

759 13. As shown in Fig. S1c, addition of magnesium ions or its combination with the

760 nonhydrolyzable ATP analog AMPPNP resulted in increases in the melting temperature

$761\left(\mathrm{~T}_{\mathrm{m}}\right)$ of CsGSIb with $\sim 6$ or $\sim 10^{\circ} \mathrm{C}$, respectively, while the presence of the substrate of 
762 glutamate showed no apparent effect on $\mathrm{T}_{\mathrm{m}}$, consistent with previous observations ${ }^{13}$. In

763 contrast, as evidenced by SEC-MALS measurements (Fig. S1d), the presence of the

764 above ligands showed no appreciable effect on the decamer-forming properties. These

765 observations collectively indicate that while binding to substrate or cofactor rigidifies the

766 structural organization within individual pentamer rings, the inter-ring assembly of GSII

767 is, at least in vitro, not substrate-induced.

768

769 
a

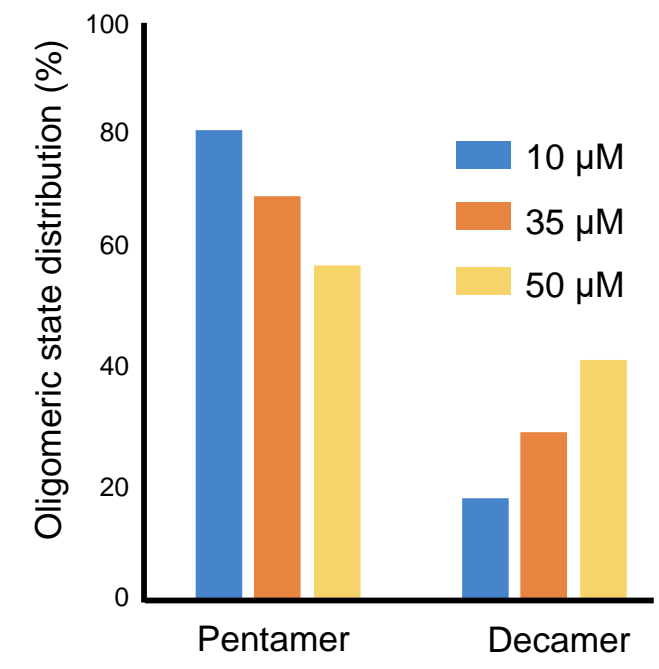

C

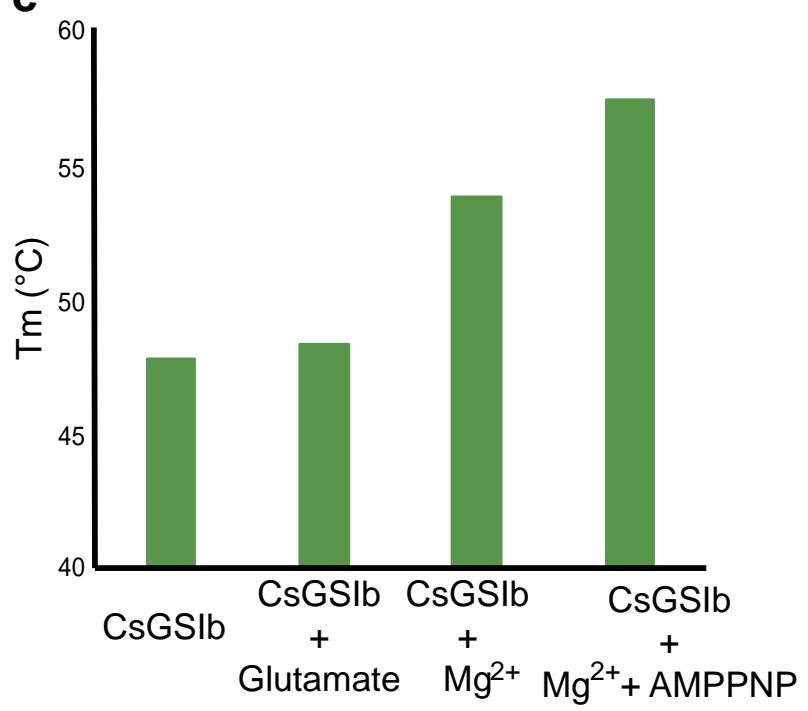

b

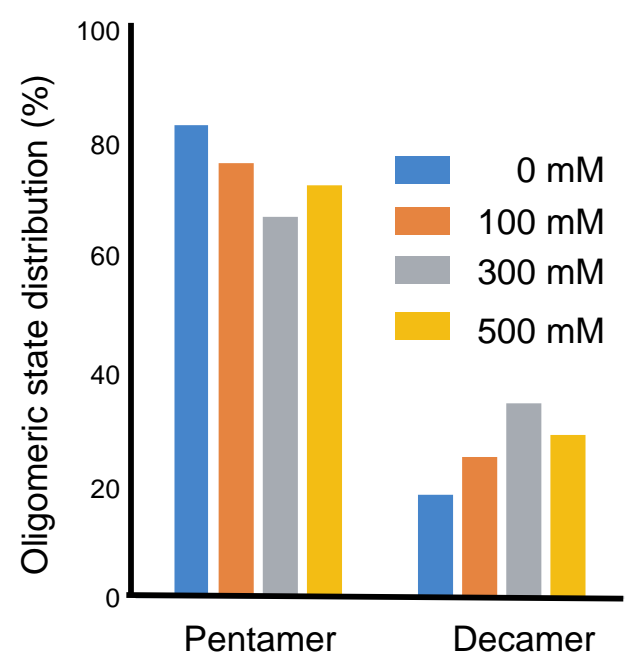

d

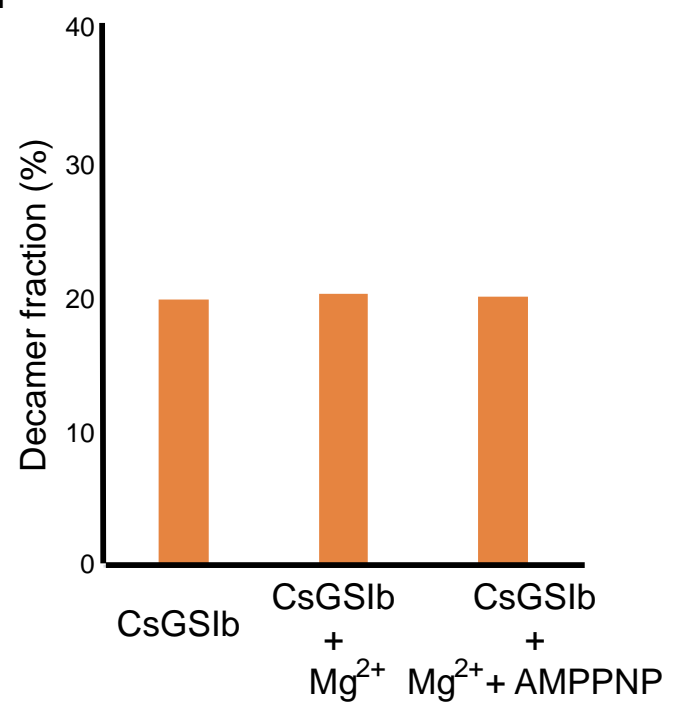

Fig. S1: CsGslb exhibits a pentamer-decamer dynamic equilibrium in solution. Using SEC-MALS to reveal the dependence of CsGslb assembly status on: a, protein concentrations; b, salt concentrations. c, Melting temperatures of CsGslb in presence of various substrates as measured by differential scanning fluorimetry. $\mathbf{d}$, Effects of various substrates on quaternary assembly status of CsGslb as measured by SEC-MALS. 

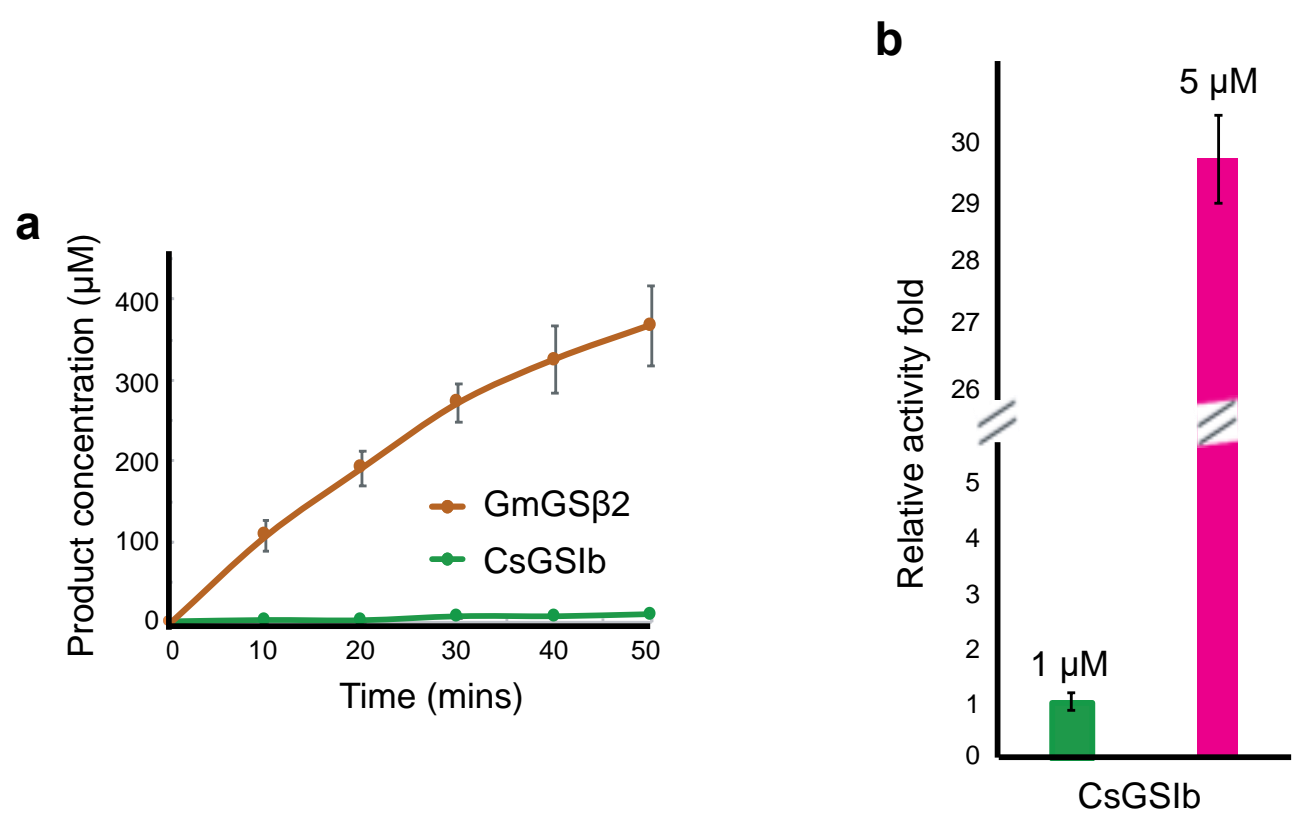

Fig. S2: Enzymatic characterizatioin of GmGS $\beta 2$ and CsGSIb.

a, Time courses of GS activity for GmGS $\beta 2$ (orange) and CsGSIb (green). b, GS activity of CsGSIb demonstrates significant concentration-dependence. Green bar: $1 \mu \mathrm{M}$ enzyme; Pink barL $5 \mu \mathrm{M}$ enzyme, Enzyme concentrations are shown as monomer concentration. Reaction conditions are same as that in Fig. 2. All experiments were repeated three times and data are shown as means \pm s.d. 
a

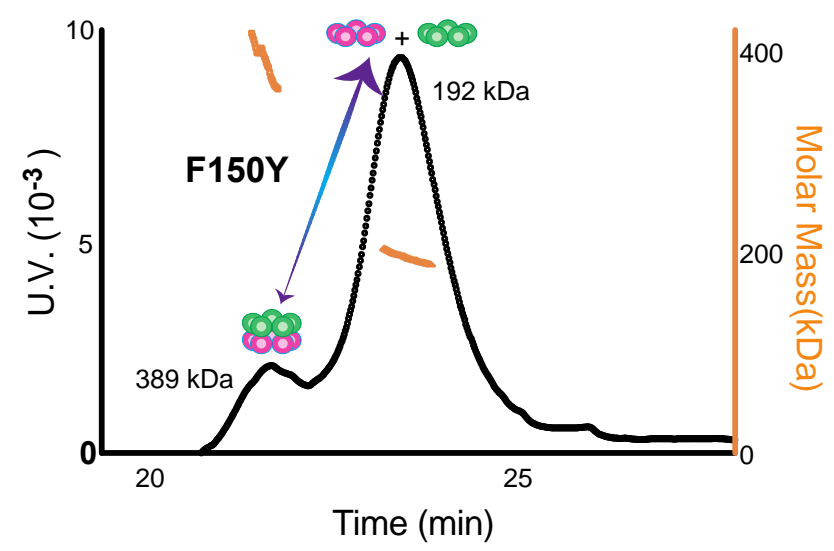

b

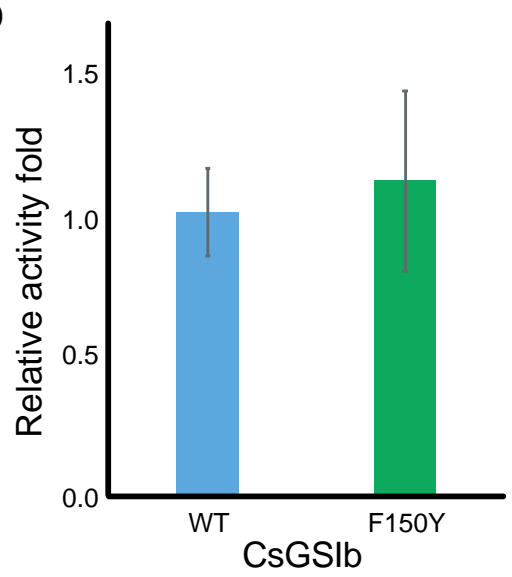

Fig. S3: Mutation effects of F150Y on the quaternary assembly property and enzyme activity of CsGslb.

a, SEC-MALS profile of CsGslb F150Y mutant reveals no change in the quaternary assembly property upon mutation. b. Wild type CsGslb and F150Y demonstrate similar GS activities. Activity assays were performed three times in the condition same as that in Fig.2. Data are shown as means \pm s.d. 
a

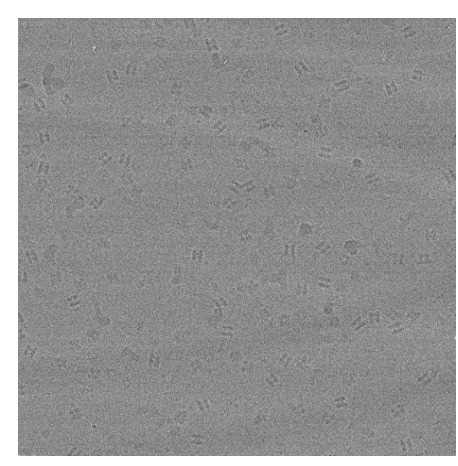

b

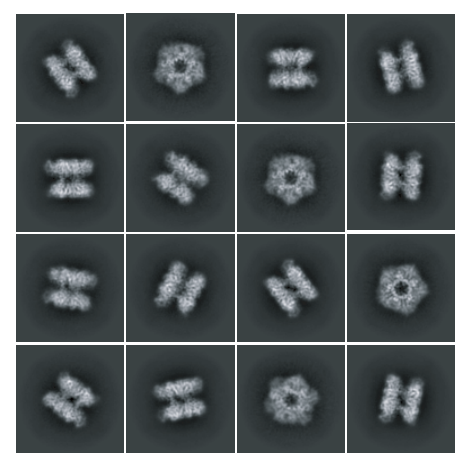

C
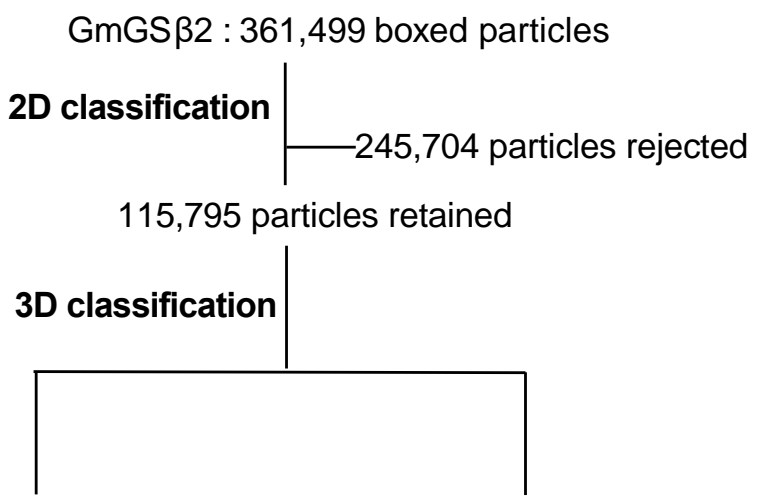

11,078 Junk particles
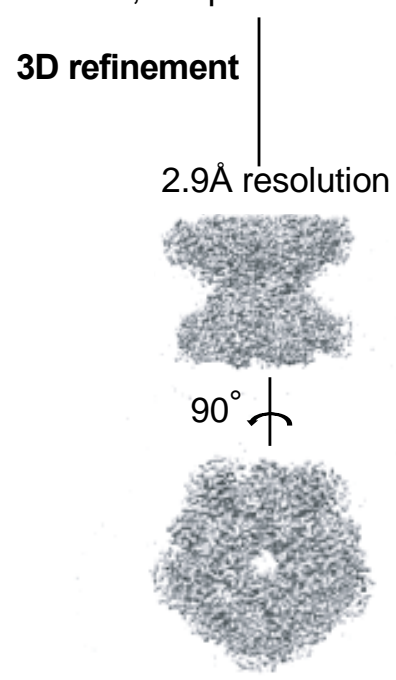

Fig. S4: Cryo-EM anaysis of GmGS $\beta 2$.

a, Representative cryo-EM micrograph; $\mathbf{b}$, Subset of representative, reference-free 2D class averages; c, Data processing workflow. 


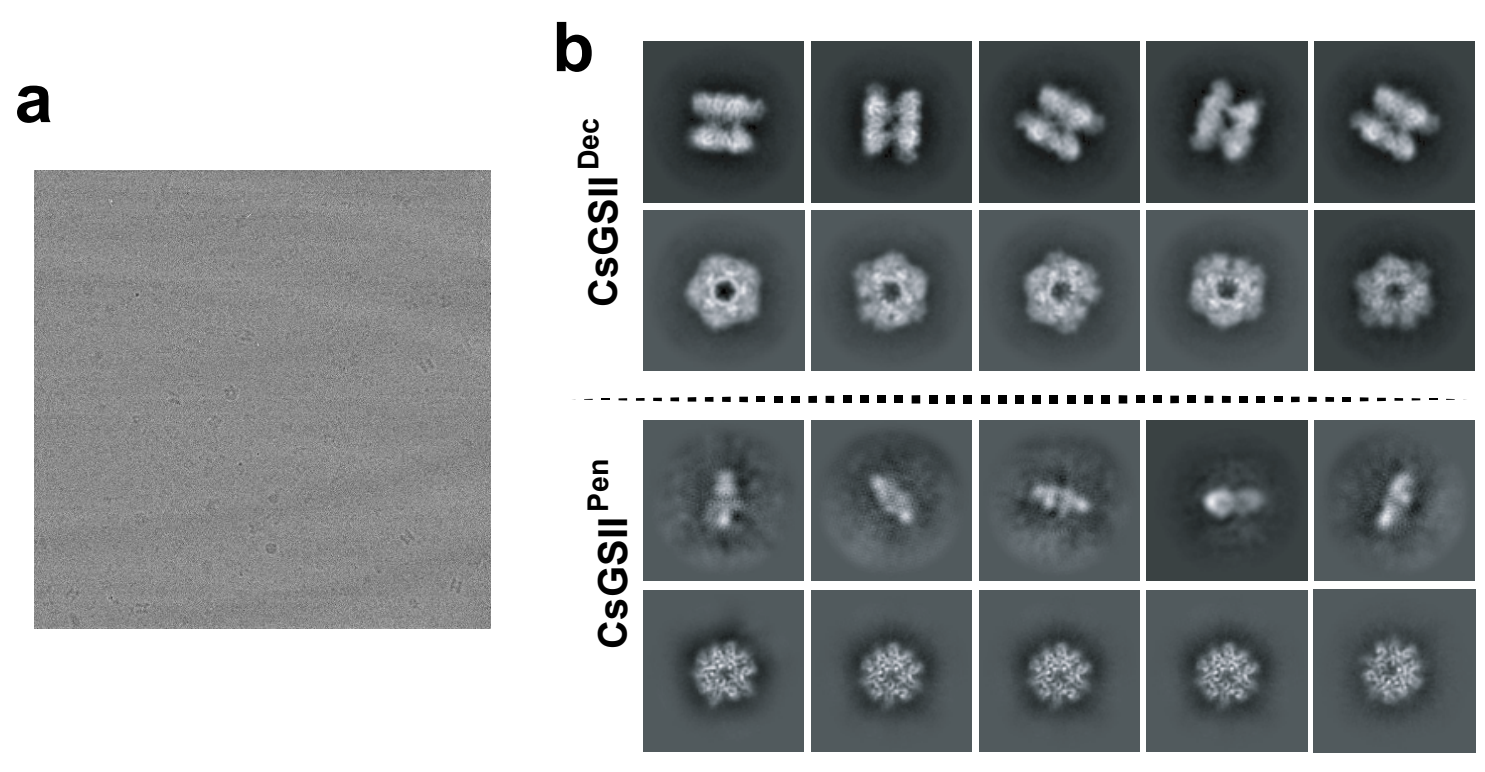

C

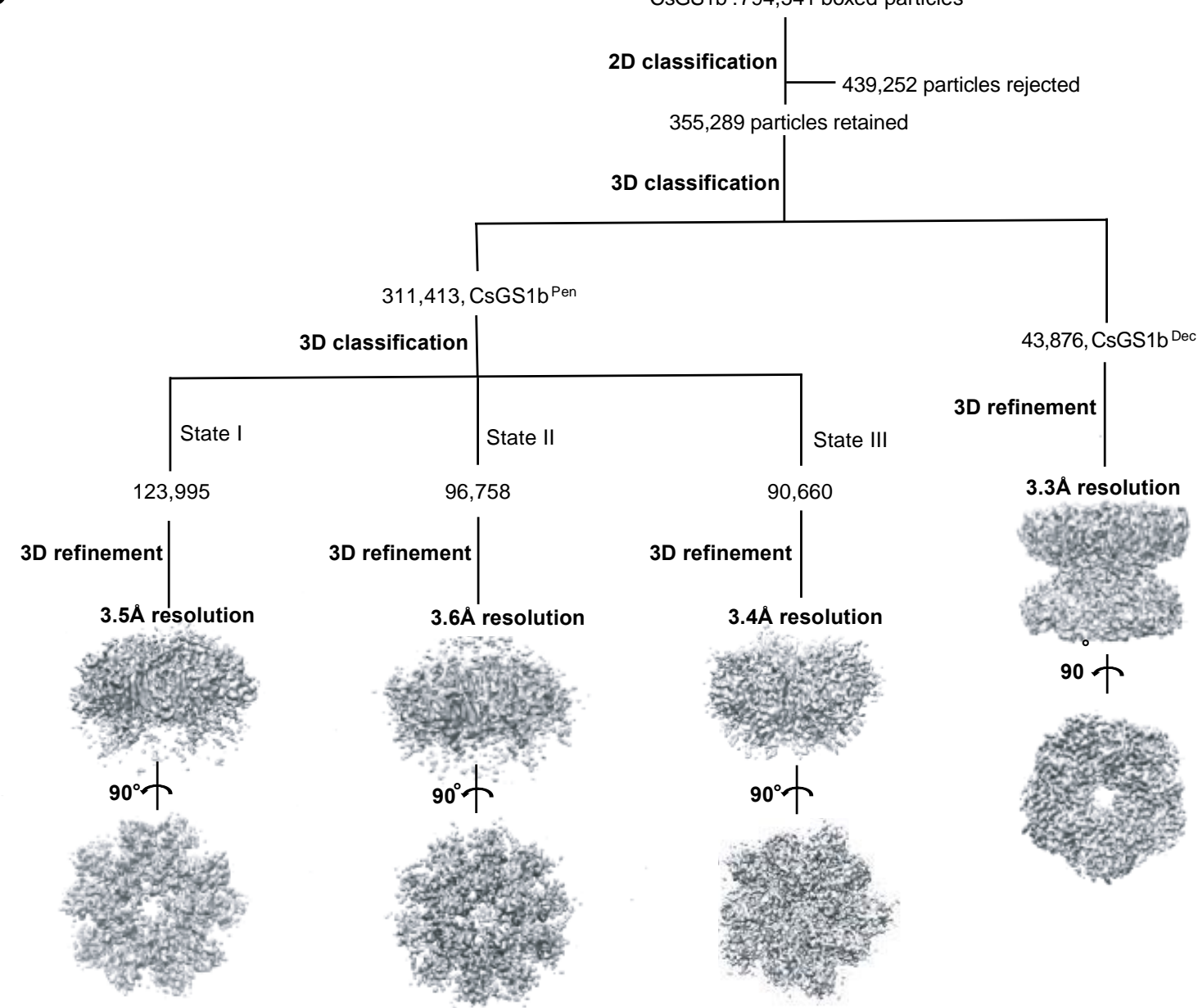

Fig. S5: Cryo-EM anaysis of CsGSIb.

a, Representative cryo-EM micrograph; $\mathbf{b}$, Subset of representative, reference-free 2D class averages; c, Data processing workflow. 
a

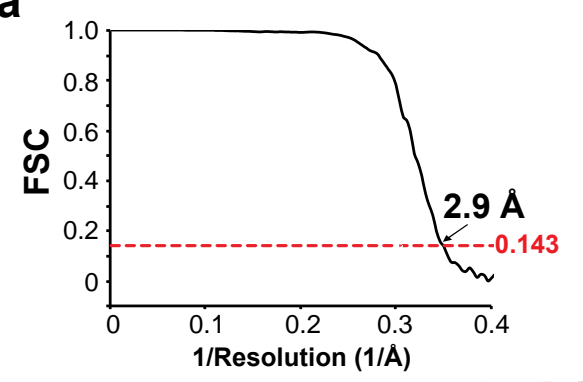

b

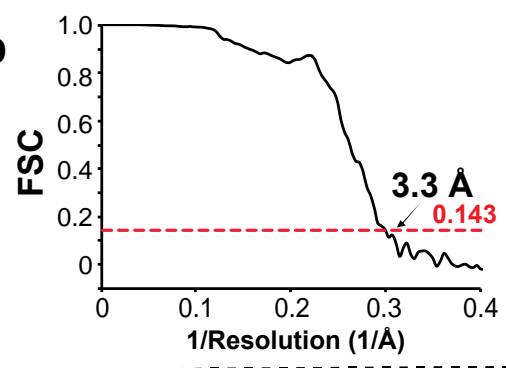

C

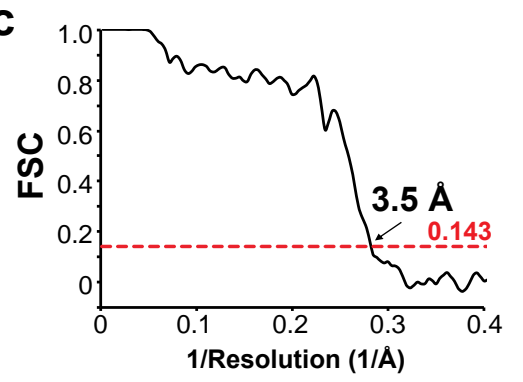

d

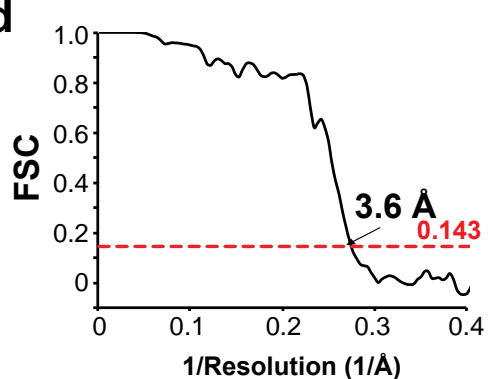

e

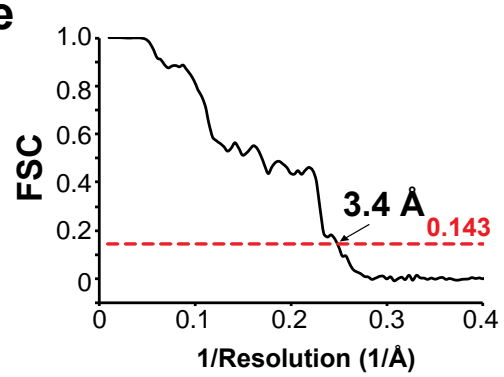

GmGS $\beta 2$
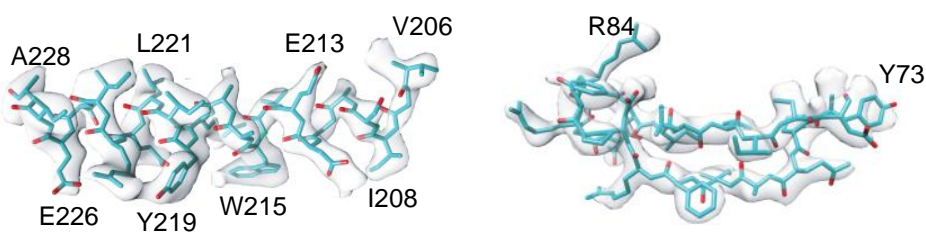

CsGSIb ${ }^{\text {Dec }}$

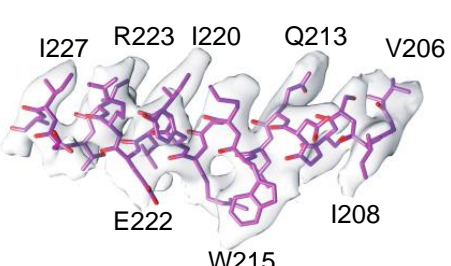

W215

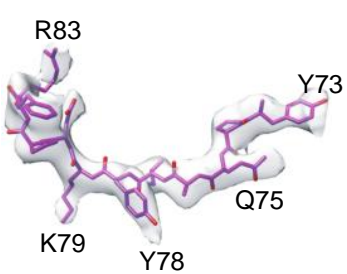

CsGSIb ${ }^{\text {Pen }}$ (state I)

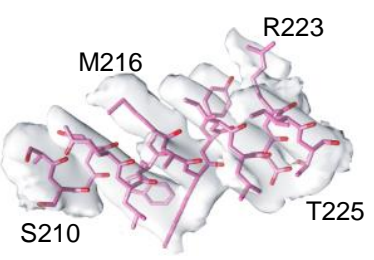

$\mathrm{R} 218$

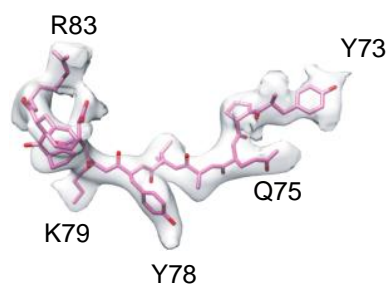

CsGSIb $^{\text {Pen }}$ (state II)
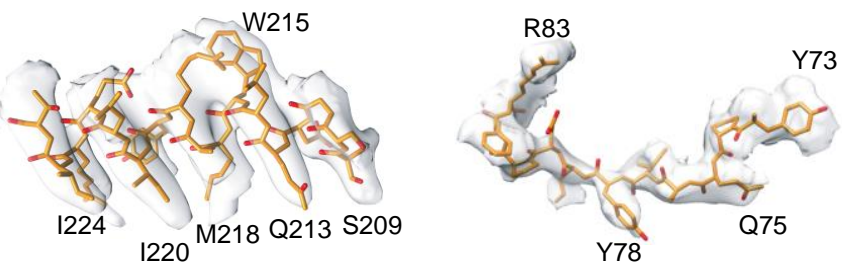

CsGSIb $^{\text {Pen }}$ (state III)
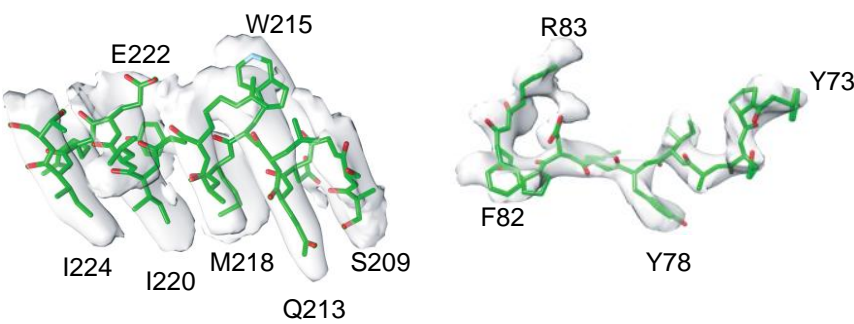

Fig. S6: Validation of cryo-EM structures

a-e: GmGS $\beta 2$, CsGSIbDec, and three states of CsGSIb ${ }^{P e n}$, respectively. Left: Gold-standard FSC plots generated from cryoSPARC; Right: View of model fitted in representative density. 
a

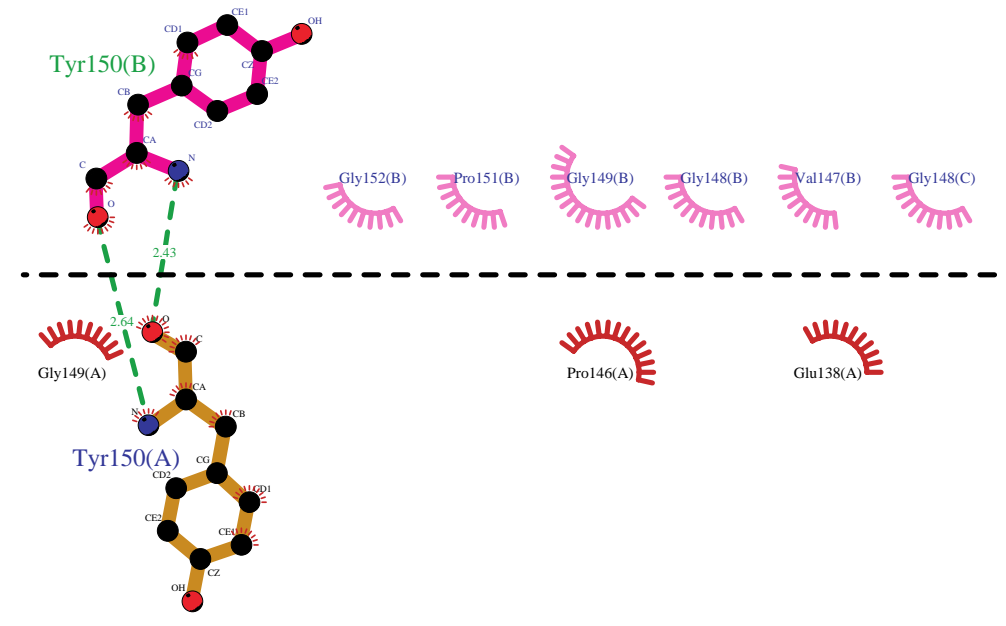

b

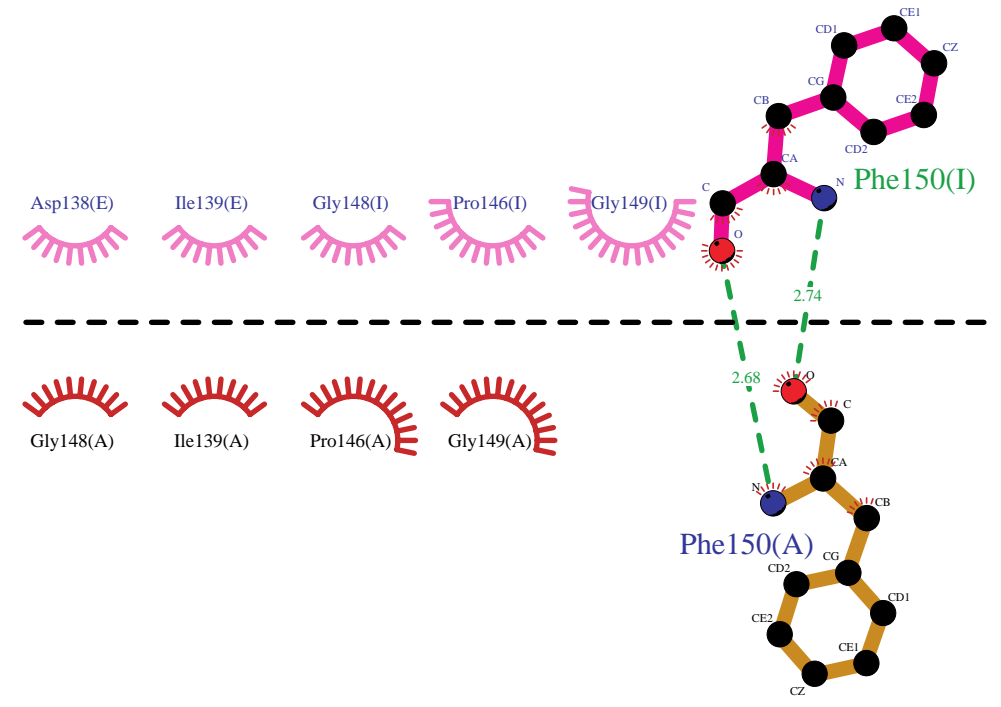

C

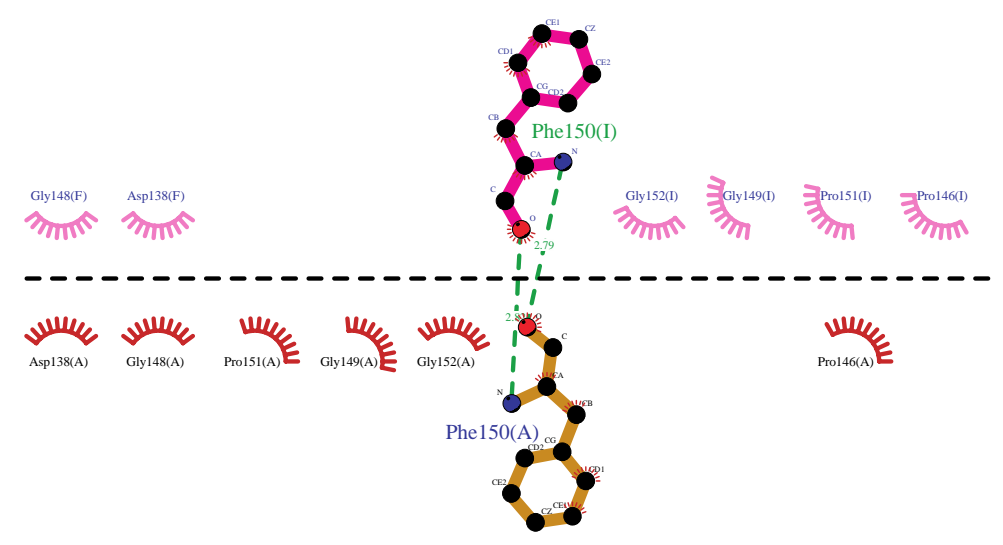

Fig. S7: Detailed analysis of interactions bewtween two GSII pentamric rings using the program of Ligplot. a, CsGSIb; b, GmGSß2; and c, Maize GS using the pdb code of 2d3a. 

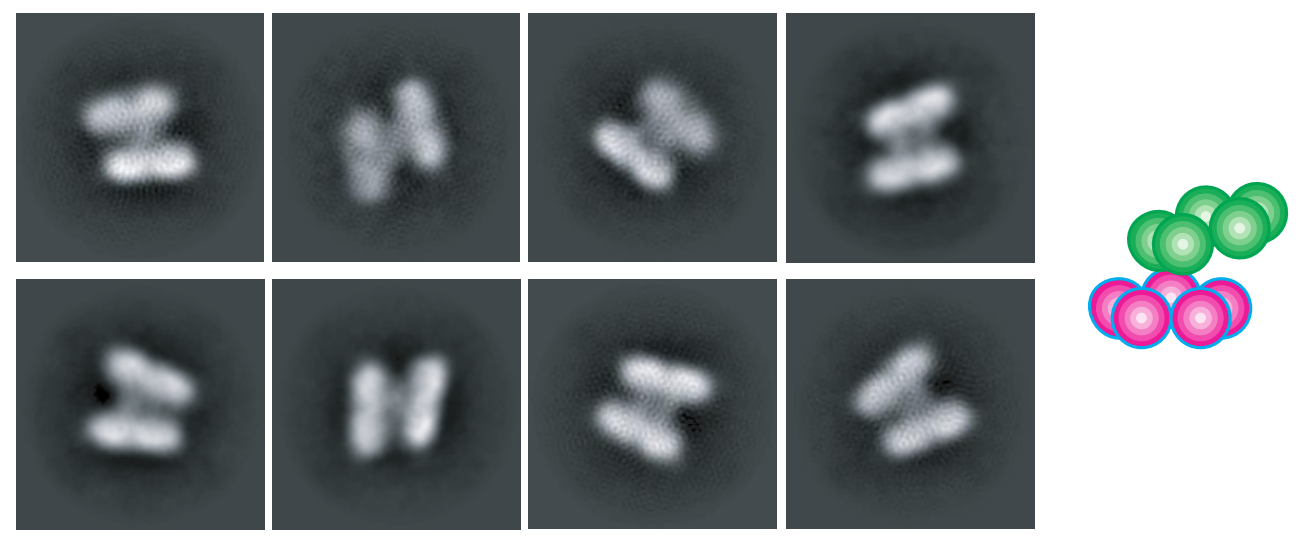

Fig. S8: Swinging motion of CsGslb rings. Two-dimensional class averages of CsGslb particles reveals a few class of particles in which two pentameric rings are no longer parallel. This swinging motion of the rings with respect to each other is likely to be owing to flexibility of the inter-ring connections. Right: A schematic representation of the averages is shown for 

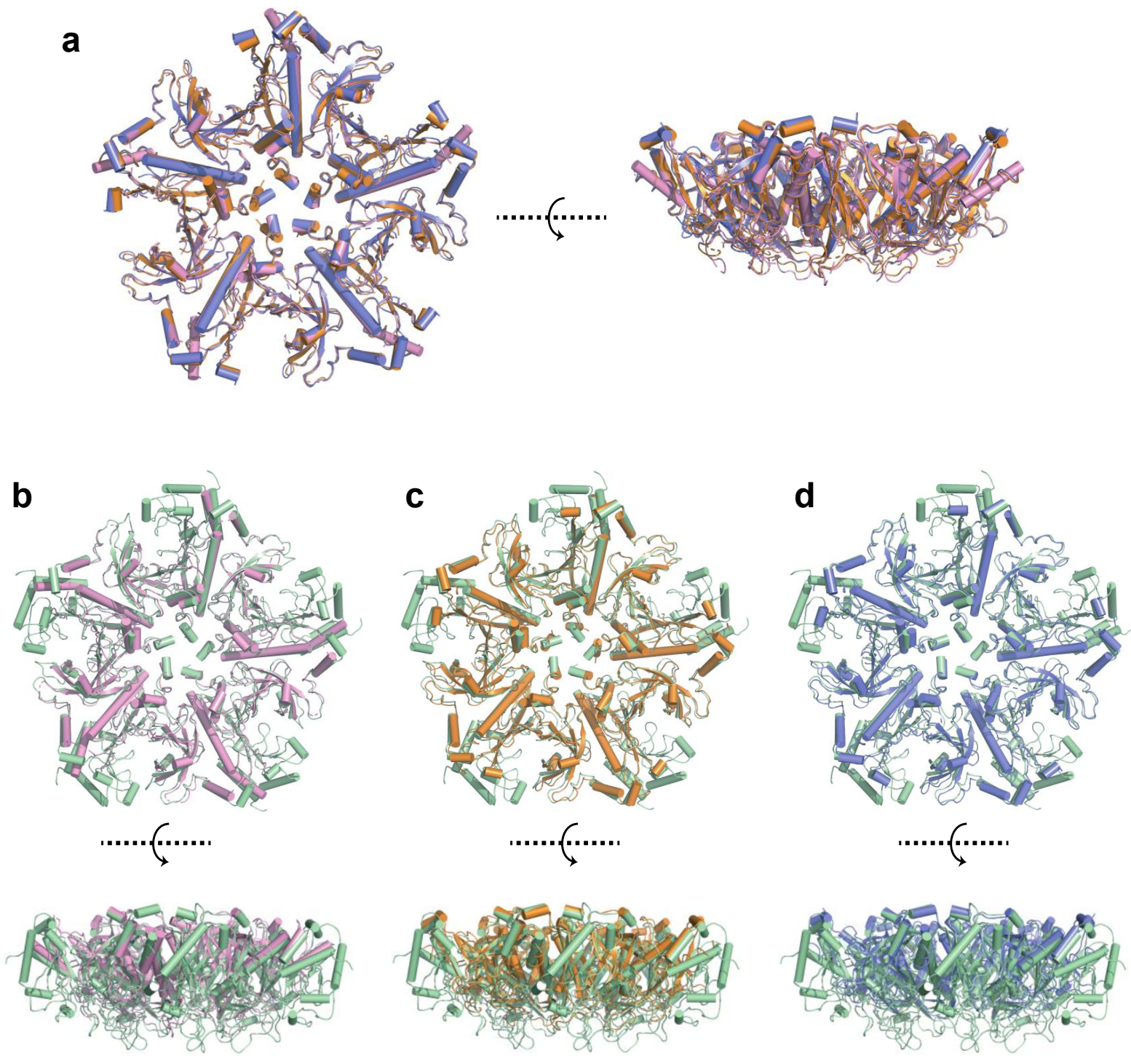

Fig. S9: Three cryo-EM structures of CsGSIb pentamers in isolation.

a, Structure allignment of three CsGSIbPen structures determined using Cryo-EM. Left: Topview; Right: Sideview. These three stuctures, colored in golden, pink and pruple, respectively, are highly similar to each other, with only a few structural variations at the peripheral regions. $\mathbf{b}$-d, Structure comparison of three structures of CsGSIb pentamer in isolation (colored the same as in a) with that in the context of decamer (in color of green). Upper: Topview; Lower: Sideview. Note a large portion is missing in the structure of CsGSIbPen arising from electron density missing, indicating those regions are highly dynamic. 


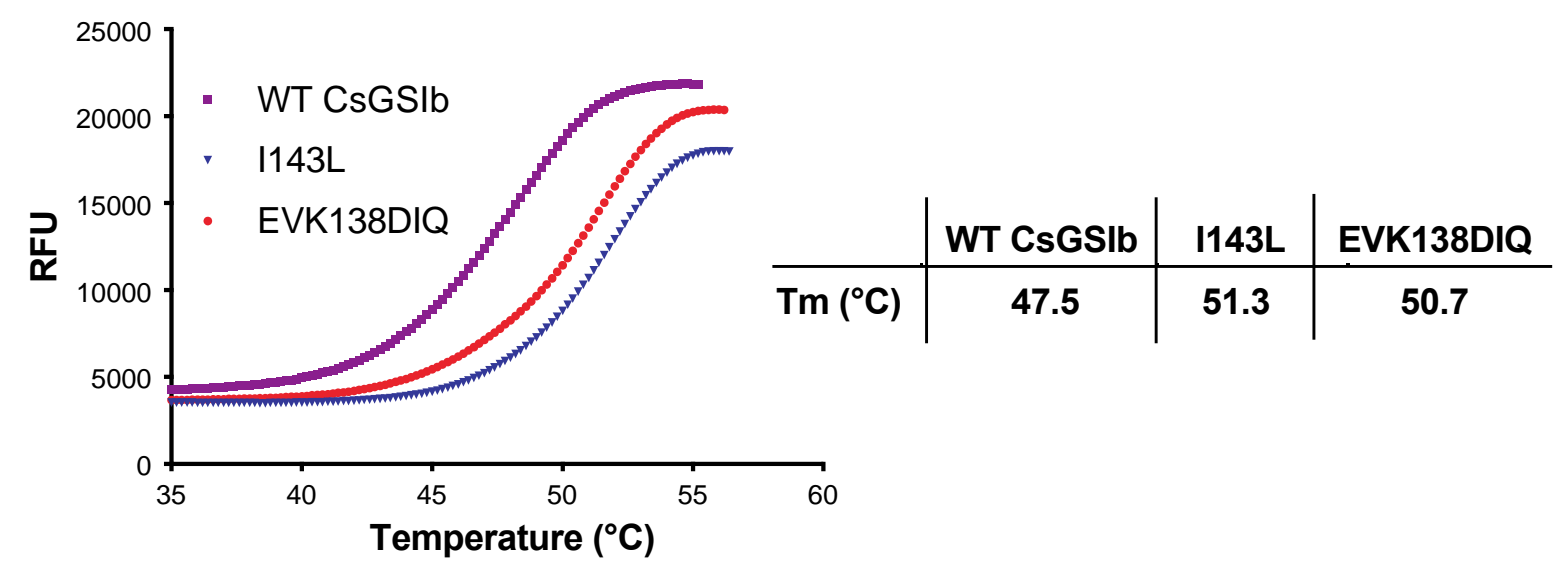

Fig. S10 Thermal shift assays for CsGSIb and mutants.

The thermal stabilities of wild type CsGsll and mutants were analysed by measuring SYPRO Orange dye fluorescence over a temperature ranging from 35 to $\sim 55^{\circ} \mathrm{C}$ using a real-time PCR thermocycler. Left: Representative unfolding curves; Right: Derived meting temperatures. RFU: Relative fluorescence unit. The lower value of meting temperature for WT CsGSIb indicates structural instablity for the wild type CsGSIb. 


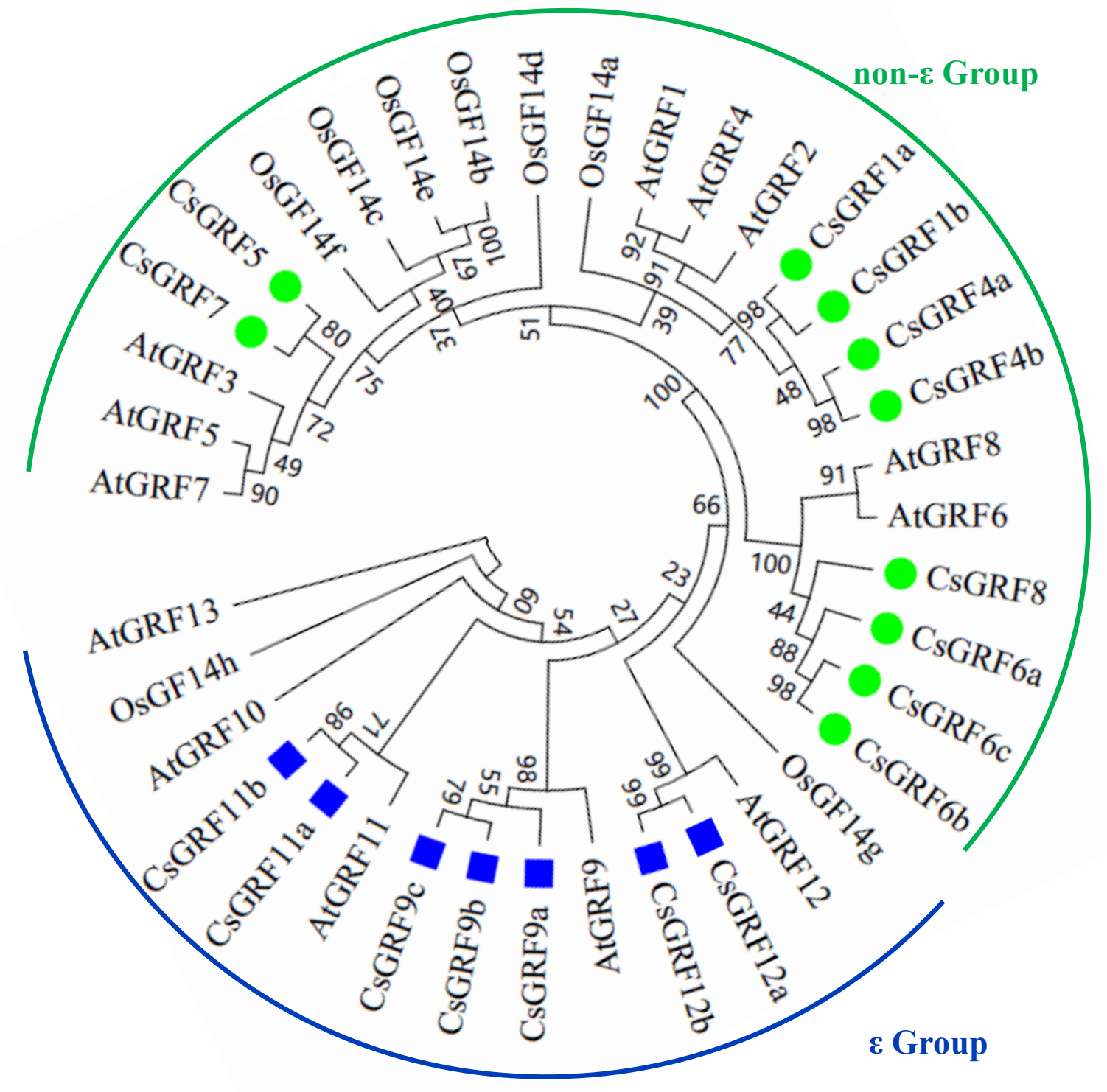

Fig. S11. Phylogenetic analysis of Cs14-3-3s from Camellia sinensis genome as compared with 14-3-3s from Arabidopsis and rice. Amino acid sequences were aligned by Clustal W. MEGA 6.0 software was used to construct the phylogenetic tree by the NJ method with 1000 bootstrap replicates. They are divided into two groups of non- $\varepsilon$ Group and $\varepsilon$ Group. 

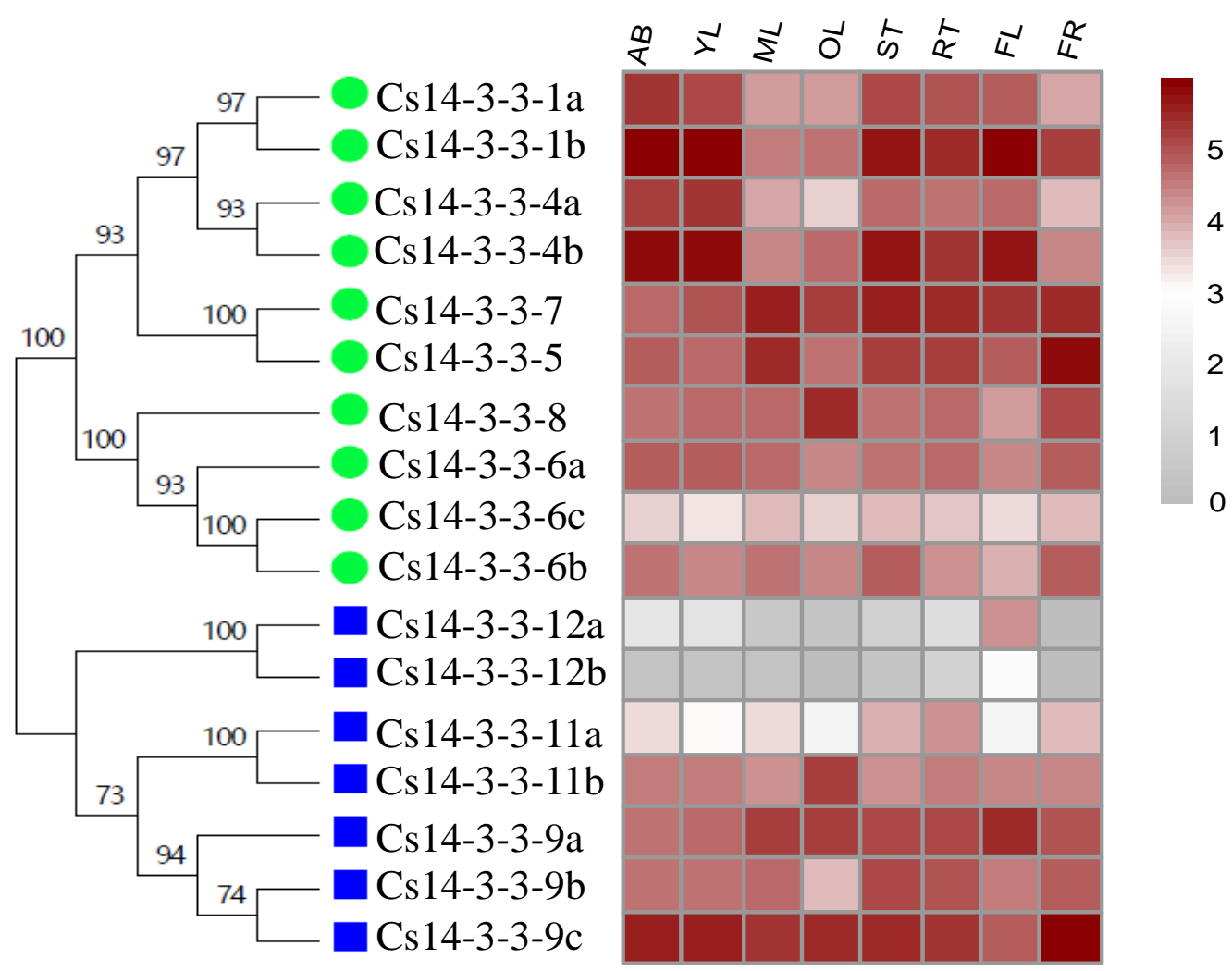

Fig. S12. Expression of Cs14-3-3 genes in eight tissues of Camellia sinensis plants., including $A B$, apical bud refers to unopened leaves on the top of activity growing shoots; $Y L$, young leaf includes the first and second leaf below the apical bud; $M L$, mature leaf is for these geminated in the spring and are harvested in the autumn; OL, old leaf for these in the bottom of tea tree plant; FL, Flower; FR, fruit of tea plants, ST, Stem for the 2nd, 3rd internodes; RT, roots, were retrived from RNA Sequencing data. Expression levels were calculated using Log10 (FPKM). 


\begin{tabular}{|c|c|c|c|c|c|}
\hline & $\begin{array}{l}\mathrm{CsGSIb}^{\mathrm{Dec}} \\
(\mathrm{PDB} 7 \mathrm{~V} 4 \mathrm{I})\end{array}$ & $\begin{array}{c}\mathrm{CsGS1b}^{\text {Pen }}(\mathrm{I}) \\
(\mathrm{PDB} 7 \mathrm{~V} 4 \mathrm{~J})\end{array}$ & $\begin{array}{l}\mathrm{CsGSIb}^{\text {Pen }}(\mathrm{II}) \\
(\mathrm{PDB} 7 \mathrm{~V} 4 \mathrm{~K})\end{array}$ & $\begin{array}{l}\mathrm{CsGSIb}^{\text {Pen }}(\mathrm{III}) \\
(\mathrm{PDB} 7 \mathrm{~V} 4 \mathrm{~L})\end{array}$ & $\begin{array}{l}\text { GmGS } \beta 2 \\
(\mathrm{PDB} 7 \mathrm{~V} 4 \mathrm{H})\end{array}$ \\
\hline \multicolumn{6}{|c|}{ Data collection } \\
\hline Magnification & $29000 x$ & $29000 x$ & $29000 x$ & $29000 x$ & $29000 x$ \\
\hline Voltage (kV) & 300 & 300 & 300 & 300 & 300 \\
\hline $\begin{array}{l}\text { Electron exposure } \\
\text { (e-/Å2) }\end{array}$ & 51 & 51 & 51 & 51 & 51 \\
\hline Defocus range $(\mu \mathrm{m})$ & $-1.6 \sim-2.3$ & $-1.6 \sim-2.3$ & $-1.6 \sim-2.3$ & $-1.6 \sim-2.3$ & $-1.6 \sim-2.3$ \\
\hline Pixel size (Å) & 0.505 & 0.505 & 0.505 & 0.505 & 0.505 \\
\hline \multicolumn{6}{|c|}{ Reconstruction and Model composition } \\
\hline Symmetry imposed & D5 & C5 & C5 & C5 & D5 \\
\hline Chains & 10 & 5 & 5 & 5 & 10 \\
\hline Nonhydrogen atoms & 27680 & 9850 & 9260 & 8966 & 27340 \\
\hline Protein residues & 3560 & 1275 & 1195 & 1140 & 3520 \\
\hline \multicolumn{6}{|c|}{ Refinement } \\
\hline \multicolumn{6}{|c|}{ R.m.s. deviations } \\
\hline Bond lengths $(\AA)$ & 0.003 & 0.003 & 0.004 & 0.004 & 0.004 \\
\hline Bond angles $\left({ }^{\circ}\right)$ & 0.585 & 0.670 & 0.660 & 0.696 & 0.589 \\
\hline Model-to-map fit (CC) & 0.74 & 0.52 & 0.64 & 0.57 & 0.82 \\
\hline Map resolution $(\AA ̊)$ & 3.3 & 3.5 & 3.6 & 3.4 & 2.9 \\
\hline FSC threshold & 0.143 & 0.143 & 0.143 & 0.143 & 0.143 \\
\hline \multicolumn{6}{|c|}{ Validation } \\
\hline MolProbity score & 1.91 & 2.28 & 1.98 & 2.24 & 1.63 \\
\hline Clashscore & 10.27 & 19.49 & 17.27 & 17.81 & 7.07 \\
\hline Rotamers outliers (\%) & 0.00 & 0.00 & 0.20 & 0.00 & 0.00 \\
\hline \multicolumn{6}{|c|}{ Ramachandran plot } \\
\hline Favored (\%) & 94.46 & 91.84 & 96.33 & 91.87 & 96.37 \\
\hline Allowed (\%) & 5.54 & 8.16 & 3.67 & 8.13 & 3.63 \\
\hline Outliers (\%) & 0.00 & 0.00 & 0.00 & 0.00 & 0.00 \\
\hline
\end{tabular}




\section{References:}

865 1. Marsh, J.A. et al. Protein complexes are under evolutionary selection to assemble via

$866 \quad$ ordered pathways. Cell 153, 461-70 (2013).

867 2. Goodsell, D.S. \& Olson, A.J. Structural symmetry and protein function. Annu Rev Biophys

$868 \quad$ Biomol Struct 29, 105-53 (2000).

869 3. Levy, E.D., Boeri Erba, E., Robinson, C.V. \& Teichmann, S.A. Assembly reflects evolution

$870 \quad$ of protein complexes. Nature 453, 1262-5 (2008).

871 4. Eisenberg, D., Gill, H.S., Pfluegl, G.M. \& Rotstein, S.H. Structure-function relationships of

872 glutamine synthetases. Biochim Biophys Acta 1477, 122-45 (2000).

873 5. Stadtman, E.R., Ginsburg, A. The glutamine synthetase of Escherichia coli: structure and

874 control. In: Boyer, P.D. (Ed.), The Enzymes, 10, 755-807 (1974).

875 6. Brown, J.R., Masuchi, Y., Robb, F.T. \& Doolittle, W.F. Evolutionary relationships of

876 bacterial and archaeal glutamine synthetase genes. J Mol Evol 38, 566-76 (1994).

877 7. van Rooyen, J.M., Abratt, V.R., Belrhali, H. \& Sewell, T. Crystal structure of Type III

878 glutamine synthetase: surprising reversal of the inter-ring interface. Structure 19, 471-

$879 \quad 83$ (2011).

880 8. Almassy, R.J., Janson, C.A., Hamlin, R., Xuong, N.H. \& Eisenberg, D. Novel subunitsubunit interactions in the structure of glutamine synthetase. Nature 323, 304-9 (1986).

9. Gill, H.S. \& Eisenberg, D. The crystal structure of phosphinothricin in the active site of glutamine synthetase illuminates the mechanism of enzymatic inhibition. Biochemistry 40, 1903-12 (2001).

10. Gill, H.S., Pfluegl, G.M. \& Eisenberg, D. Multicopy crystallographic refinement of a relaxed glutamine synthetase from Mycobacterium tuberculosis highlights flexible loops in the enzymatic mechanism and its regulation. Biochemistry 41, 9863-72 (2002).

11. Unno, H. et al. Atomic structure of plant glutamine synthetase: a key enzyme for plant productivity. J Biol Chem 281, 29287-96 (2006).

12. Torreira, E. et al. The structures of cytosolic and plastid-located glutamine synthetases from Medicago truncatula reveal a common and dynamic architecture. Acta Crystallogr D Biol Crystallogr 70, 981-93 (2014).

13. Krajewski, W.W. et al. Crystal structures of mammalian glutamine synthetases illustrate substrate-induced conformational changes and provide opportunities for drug and herbicide design. J Mol Biol 375, 217-28 (2008).

14. Betti, M. et al. Glutamine synthetase in legumes: recent advances in enzyme structure and functional genomics. Int J Mol Sci 13, 7994-8024 (2012).

15. Llorca, O. et al. The three-dimensional structure of an eukaryotic glutamine synthetase: functional implications of its oligomeric structure. J Struct Biol 156, 469-79 (2006).

16. Mack, G. Glutamine synthetase isoenzymes, oligomers and subunits from hairy roots of Beta vulgaris L. var. lutea. Planta 205, 113-20 (1998).

17. Armache, J.P. \& Cheng, Y. Single-particle cryo-EM: beyond the resolution. Nat/ Sci Rev 6, 864-866 (2019).

18. Tzivion, G., Shen, Y.H. \& Zhu, J. 14-3-3 proteins; bringing new definitions to scaffolding. Oncogene 20, 6331-8 (2001). 
906

907

908

909

910

911

912

913

914

915

916

917

918

919

920

921

922

923

924

925

926

927

928

929

930

931

932

933

934

935

936

937

938

939

940

941

942

943

944

945

946

947

948

949

19. Chevalier, D., Morris, E.R. \& Walker, J.C. 14-3-3 and FHA domains mediate phosphoprotein interactions. Annu Rev Plant Biol 60, 67-91 (2009).

20. Obsilova, V. \& Obsil, T. The 14-3-3 Proteins as Important Allosteric Regulators of Protein Kinases. Int J Mol Sci 21(2020).

21. Kondo, Y. et al. Cryo-EM structure of a dimeric B-Raf:14-3-3 complex reveals asymmetry in the active sites of B-Raf kinases. Science 366, 109-115 (2019).

22. Finnemann, J. \& Schjoerring, J.K. Post-translational regulation of cytosolic glutamine synthetase by reversible phosphorylation and 14-3-3 protein interaction. Plant J 24, 17181 (2000).

23. Pozuelo, M., MacKintosh, C., Galvan, A. \& Fernandez, E. Cytosolic glutamine synthetase and not nitrate reductase from the green alga Chlamydomonas reinhardtii is phosphorylated and binds 14-3-3 proteins. Planta 212, 264-9 (2001).

24. Lima, L., Seabra, A., Melo, P., Cullimore, J. \& Carvalho, H. Phosphorylation and subsequent interaction with 14-3-3 proteins regulate plastid glutamine synthetase in Medicago truncatula. Planta 223, 558-67 (2006).

25. Riedel, J., Tischner, R. \& Mack, G. The chloroplastic glutamine synthetase (GS-2) of tobacco is phosphorylated and associated with 14-3-3 proteins inside the chloroplast. Planta 213, 396-401 (2001).

26. Kerppola, T.K. Design and implementation of bimolecular fluorescence complementation (BiFC) assays for the visualization of protein interactions in living cells. Nat Protoc 1, 1278-86 (2006).

27. Kuriyan, J. \& Eisenberg, D. The origin of protein interactions and allostery in colocalization. Nature 450, 983-90 (2007).

28. Huang, Z. et al. ASD: a comprehensive database of allosteric proteins and modulators. Nucleic Acids Res 39, D663-9 (2011).

29. Red Brewer, M. et al. Mechanism for activation of mutated epidermal growth factor receptors in lung cancer. Proc Natl Acad Sci U S A 110, E3595-604 (2013).

30. Capdevila, D.A., Braymer, J.J., Edmonds, K.A., Wu, H. \& Giedroc, D.P. Entropy redistribution controls allostery in a metalloregulatory protein. Proc Natl Acad Sci U SA 114, 4424-4429 (2017).

31. Tzeng, S.R. \& Kalodimos, C.G. Dynamic activation of an allosteric regulatory protein. Nature 462, 368-72 (2009).

32. Tzeng, S.R. \& Kalodimos, C.G. Protein activity regulation by conformational entropy. Nature 488, 236-40 (2012).

33. Veglia, G. \& Cembran, A. Role of conformational entropy in the activity and regulation of the catalytic subunit of protein kinase A. FEBS J 280, 5608-15 (2013).

34. Bernard, S.M. \& Habash, D.Z. The importance of cytosolic glutamine synthetase in nitrogen assimilation and recycling. New Phytol 182, 608-20 (2009).

35. Montanini, B. et al. Distinctive properties and expression profiles of glutamine synthetase from a plant symbiotic fungus. Biochem J 373, 357-68 (2003).

36. Sakakibara, H. et al. Molecular identification and characterization of cytosolic isoforms of glutamine synthetase in maize roots. J Biol Chem 271, 29561-8 (1996).

37. Seabra, A.R. \& Carvalho, H.G. Glutamine synthetase in Medicago truncatula, unveiling new secrets of a very old enzyme. Front Plant Sci 6, 578 (2015). 
950 38. Denman, R.B. \& Wedler, F.C. Association-dissociation of mammalian brain glutamine (1984).

954

955

956

957

958

959

960

961

962

963

964

965

966

967

39. Mora, J. Glutamine metabolism and cycling in Neurospora crassa. Microbiol Rev 54, 293304 (1990).

40. Mowbray, S.L., Kathiravan, M.K., Pandey, A.A. \& Odell, L.R. Inhibition of glutamine synthetase: a potential drug target in Mycobacterium tuberculosis. Molecules 19, 13161-76 (2014).

41. Thomsen, H.C., Eriksson, D., Moller, I.S. \& Schjoerring, J.K. Cytosolic glutamine synthetase: a target for improvement of crop nitrogen use efficiency? Trends Plant Sci 19, 656-63 (2014).

42. Harth, G. \& Horwitz, M.A. Inhibition of Mycobacterium tuberculosis glutamine synthetase as a novel antibiotic strategy against tuberculosis: demonstration of efficacy in vivo. Infect Immun 71, 456-64 (2003).

43. Gising, J. et al. Trisubstituted imidazoles as Mycobacterium tuberculosis glutamine synthetase inhibitors. J Med Chem 55, 2894-8 (2012).

44. Kumari, M. \& Subbarao, N. Virtual screening to identify novel potential inhibitors for Glutamine synthetase of Mycobacterium tuberculosis. J Biomol Struct Dyn 38, 50625080 (2020).

45. Gawronski, J.D. \& Benson, D.R. Microtiter assay for glutamine synthetase biosynthetic activity using inorganic phosphate detection. Anal Biochem 327, 114-8 (2004).

46. Masalkar, P.D. \& Roberts, D.M. Glutamine synthetase isoforms in nitrogen-fixing soybean nodules: distinct oligomeric structures and thiol-based regulation. FEBS Lett 589, 215-21 (2015).

47. Punjani, A., Rubinstein, J.L., Fleet, D.J. \& Brubaker, M.A. cryoSPARC: algorithms for rapid unsupervised cryo-EM structure determination. Nat Methods 14, 290-296 (2017).

48. Yang, J. \& Zhang, Y. I-TASSER server: new development for protein structure and function predictions. Nucleic Acids Res 43, W174-81 (2015).

49. Pettersen, E.F. et al. UCSF Chimera--a visualization system for exploratory research and analysis. J Comput Chem 25, 1605-12 (2004).

50. Emsley, P., Lohkamp, B., Scott, W.G. \& Cowtan, K. Features and development of Coot. Acta Crystallogr D Biol Crystallogr 66, 486-501 (2010).

51. Afonine, P.V. et al. Real-space refinement in PHENIX for cryo-EM and crystallography. Acta Crystallogr D Struct Biol 74, 531-544 (2018).

52. Zhao, J. et al. MATE2 mediates vacuolar sequestration of flavonoid glycosides and glycoside malonates in Medicago truncatula. Plant Cell 23, 1536-55 (2011).

53. Lu, M. et al. Significantly increased amino acid accumulation in a novel albino branch of the tea plant (Camellia sinensis). Planta 249, 363-376 (2019).

54. Husted, S., Mattsson, M., Mollers, C., Wallbraun, M. \& Schjoerring, J.K. Photorespiratory $\mathrm{NH}(4)(+)$ production in leaves of wild-type and glutamine synthetase 2 antisense oilseed rape. Plant Physiol 130, 989-98 (2002). 